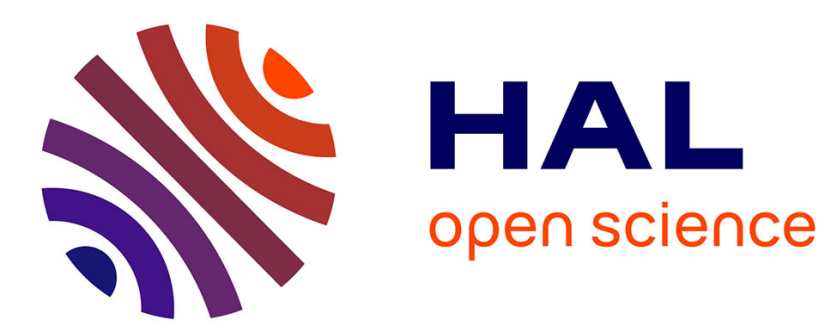

\title{
Études sur le moment et le champ moléculaire des ferromagnétiques
}

Louis Néel

\section{To cite this version:}

Louis Néel. Études sur le moment et le champ moléculaire des ferromagnétiques. Annales De Physique, 1937, 11 (8), pp.237-308. 10.1051/anphys/193711080237 . hal-02888366

\section{HAL Id: hal-02888366 \\ https://hal.science/hal-02888366}

Submitted on 29 Jul 2020

HAL is a multi-disciplinary open access archive for the deposit and dissemination of scientific research documents, whether they are published or not. The documents may come from teaching and research institutions in France or abroad, or from public or private research centers.
L'archive ouverte pluridisciplinaire HAL, est destinée au dépôt et à la diffusion de documents scientifiques de niveau recherche, publiés ou non, émanant des établissements d'enseignement et de recherche français ou étrangers, des laboratoires publics ou privés. 


\title{
ÉTUDES SUR LE MOMENT \\ ET LE CHAMP MOLÉCULAIRE DES FERROMAGNÉTIQUES
}

\author{
Par M. Louis NÉEL.
}

SOMmaire. - Ire et IIe Parties. - L'auteur développe une théorie générale des anomalies de volume des ferromagnétiques: l'anomalie est la somme de deux termes, l'un, déjà connu, est indépendant de la température, l'autre est proportionnel à la température : il provient de la valeur anormale du coefficient de dilatation des ferromagnétiques. On obtient ainsi théoriquement une formule qui coïncide avec la formule trouvée autrefois par Chevenard pour représenter avec précision les résultats expérimentaux.

Les forces magnétiques entre les atomes ont été introduites sous la forme d'une énergie d'interaction entre atomes voisins, fonction de la distance de ces atomes. Des travaux antérieurs, basés sur la comparaison des propriétés magnétiques des métaux, ont permis à l'auteur de préciser cette dépendance et la présente théorie confirme ces résultats : les données expérimentales sur les anomalies de volume fournissent la dérivée première de l'énergie d'interaction par rapport à la distance ainsi qu'une relation entre la dérivée seconde et la dérivće troisième.

$I I I$ e Partie. - Deux sources entièrement indépendantes permettent ainsi de déterminer les variations de l'énergie d'interaction avec la distance des atomes. Le problème important de la variation du champ moléculaire avec la température devient abordable et a été résolu. Deux causes modifient l'énergie d'interaction : $a$ ) un effet de dilatation dù à l'augmentation progressive, par élévation de température, de la distance mutuelle des atomes interagissants $; b$ ) un effet d'oscillation : l'agitation thermique écarte et rapproche les atomes d'où modification de l'énergie moyenne. Finalement, on trouve que le champ moléculaire est une fonction linéaire de la tem pérature calculable approximativement d'après les données obtenues précédemment. Au-dessus du Ann. de Phys, $\mathrm{I}_{\mathrm{I}}^{\mathrm{e}}$ série, t. 8 (Octobre $\mathrm{1}^{3}{ }^{3} 7$ ). 
point de Curie, l'inverse de la susceptibilité d'un paramagnétique à champ moléculaire est toujours une fonction linéaire de la température, mais le coefficieut qui joue le rôle de constante de Curie n'est plus égal à la constante de Curie du paramagnétique pur correspondant. La théorie proposée permet de calculer cette dernière. C'est ainsi qu'on trouve que les constantes de Curie vraies du nickel et du cobalt sont celles qui correspondent respectivement ì 1 et 2 spin non compensés par atome.

IVe Partie. - Pour expliquer la différence qui sépare les moments ferromagnétiques des moments paramagnétiques, l'auteur suppose que l'aimantation à saturation ne correspond pas à l'orientation complète de tous les atomes porteurs de moment: certains d'entre eux seraient soustraits au champ moléculaire. Les liaisons magnétiques entre atomes voisins, créatrices du champ moléculaire, n'auraient pas une existence permanente mais discontinue el soumise à des lois statistiques. A un certain moment, un atome donoé peut ne posséder aucune liaison magnétique avec son entourage : il n'est pas orienté. La probabilité a priori d'existence d'une liaison magnétique, sup. posée constante pour chaque type de réseau cristallin, est déterminée : par exemple, en supposant connu le moment du nickel, dans le cas du système du cube à faces centrées. Les moments des autres métaux ferromagnétiques et de leurs alliages sont alors complètement calculables. L'accord entre les moments calculés et les moments observés est remarquable. De plus, on interprète ainsi d'une manière satisfaisante les courbes compliquées des moments atomiques des ferro-nickels et des ferrocobalts, en fonction de la concentration.

PREMIÈRE PARTIE

\section{Théorie des anomalies de dilatation des ferromagnétiques.}

§1. Définition des anomalies de longueur. - L'étude des substances ferromagnétiques conduit à distinguer l'aimantation spontanée $\left({ }^{1}\right)$ - c'est-à-dire l'aimantation à l'intérieur

(') Ne pas confondre aimantation spontanée et aimantation rémanente. 
d'un domaine élémentaire - de l'aimantation globale, résultante géométrique des aimantations spontanées des différents domaines élémentaires ( ${ }^{1}$ ).

La plus grande partie de l'énergie magnétique dépend de l'aimantation spontanée : pour une mème aimantation spontanée, la chaleur spécifique vraie ne varie que très peu quand varie l'aimantation globale tandis qu'on met en évidence des anomalies importantes en fonction de l'aimantation spontanée.

De même, les variations relatives de longueur, en fonction de l'aimantation globale et de sa direction par rapport aux axes eristallins, sont faibles, de l'ordre de $10^{-6}$. Je laisserai de còté ces phénomènes qui constituent la magnétostriction proprement dite. Au contraire, le: anomalies de longueur, due a a laimantat on spontanée, sont beaucoup plus grandes, de l'ordre de $10^{-3}$.

Définition de lanomalie de longueur. - Au-dessus du point de Curie, l'aimantation spontanée est nulle, ce qui permet de déterminer la loi de dilatation normale de la substance en fonction de la température et de calculer par extrapolation, au-dessous du point de Curie, la longueur $l_{\mathrm{A}}$ qu'aurait la substance à une température $t$, pour une aimantation spontànée nulle. Soit $l_{\mathrm{B}}$ la longueur réelle, à la mème température, pour une aimantation globale nulle, j'appellerai anomalie de longueur, la différence :

$$
\delta l=l_{\mathrm{B}}-l_{\Lambda} .
$$

On définit de même, l'anomalie de volume et l'anomalie de dilatation.

\$2. Origine des anomalies de longueur. - Ce sont les actions mutuelles entre les porteurs de moment qui produisent les anomalies de longueur. Dans un ferromagnétique, tel que le nickel, l'aimantation n'est due qu'au spin et le champ

(1) Cf. Werss et Foex. Le magnétisme. Paris, Armand Colin. 
moléculaire provient des interactions entre spin. L'énergie correspondante est proportionnelle au carré de l'aimantation spontanée $\sigma$. A ces actions, il faut ajouter les forces de couplage entre spin et orbite ainsi que les forces dues au champ magnétique des spin : termes de dipôles, de quadrupôles, etc. Ces dernières forces jouent un rôle prépondérant dans les phénomènes d'aimantation globale, l'hystérésis, les propriétés magnétocristallines et la magnétostriction correspondante, mais n'ont qu'une influence subordonnée sur l'aimantation spontanée.

Ainsi, les anomalies de longueur d'une substance ferromagnétique, placée dans un champ extérieur nul, non aimantée globalement, isotrope en moyenne par suite d'une cristallisation confuse, pourront être attribuées presqu'exclusivement aux seules actions entre spin, comme la déjà fait remarquer Powell (1).

Les anomalies de longueur dépendent des variations de l'énergie magnétique en fonction du volume ou, d'une façon plus expressive, des variations, en fonction de la distance des atomes, des forces de couplage entre spin. De nombreux auteurs ont déjà attiré l'attention sur la signification théorique de ces anomalies. Bauer $\left({ }^{2}\right)$ a calculé l'anomalie de longueur au zéro absolu, en se plaçant à un point de vue purement thermodynamique et en supposant que le coefficient du champ moléculaire était inversement proportionnel au volume. Avec cette hypothèse, Verschaffelt $\left({ }^{3}\right)$ calcule l'anomalie de dilatation au point de Curie sans d'ailleurs tenter - la moindre comparaison avec l'expérience. Bauer $\left({ }^{4}\right)$ luimême a reconnu que l'anomalie du nickel s'interprétait mieux avec un champ moléculaire inversement proportionnel

(') F. C Powell. Proc. Phys. Soc., 42, part. 5, (1930), 390.

( $\left.{ }^{2}\right) J$. de Phys., [6], 10 (1929), 345.

(3) Conseil Solvay, 193o. Paris, Gauthier-Villars ( 1932 ), 370.

(4) Id., 368. 
à la racine carrée du volume. Enfin, Fowler et Kapitza $\left(^{1}\right)$, Powell $\left({ }^{2}\right)$ ont fait des calculs analogues, en se plaçant au point de vue de la théorie quantique du ferromagnétisme. D'une manière générale, l'accord n'est que fragmentaireentre ces théories et l'expérience. En effet, les expériences de Chevenard $\left({ }^{3}\right)$ sur les anomalies de dilatation des ferromagnétiques et de leurs alliages, qui constituent une étude systématique de la question, fournissent le résultat fondamental suivant : l'anomalie relative de longueur $\frac{\delta l}{l}$ s'exprime en fonction de la température $\mathrm{T}$ et de l'aimantation spontanée $\sigma$ par la formule :

$$
\frac{\delta l}{l}=\sigma^{2}\left(\lambda+\mu \mathrm{T}+\nu \mathrm{T}^{2}\right) .
$$

Les théories précédentes ne donnent que le terme en $\lambda$, indépendant de la température $\left({ }^{4}\right)$; il faut donc les compléter et les préciser. Ceci semble possible depuis que l'étude comparée des propriétés magnétiques d'un assez grand nombre de substances a permis de préciser $\left(^{5}\right)$ la courbe qui représente l'énergie d'interaction, en fonction de la distance des atomes.

Je montrerai qu'à l'anomalie de volume au zéro absolu il faut ajouter une anomalie thermique essentiellement distincte qui vient de la différence des coefficients de dilatation entre une substance magnétique et la même substance supposée privée de son aimantation spontanée.

(1) Proc. Roy. Soc., 124 (1929), 1.

() Loc. cil.

(3) Pour l'exposé des travaux de Chevenard sur cette question et une bibliographie complète, consulter le rapport de Weiss au Conseil Solvay de 1930. L'anomalie de volume des ferromagnétiques. Paris, Gauthier-Villars ( $\left.19^{32}\right)$.

(4) Chevenard, déjà, avait montré la proportionnalité des variations de longueur au carré de l'aimantation spontanée par un raisonnement assez général.

(5) Néel. Annales de Physique, 5 (1936), 232. 
Au préalable, je vais calculer l'énergie élastique et l'énergie magnétique d'une substance en fonction d'une déformation isotrope et en déduire le coefficient de dilatation.

§ 3. Energie en fonction d'une déformation élastique isotrope. - L'énergie potentielle est la somme d'une énergie élastique $W_{E}$ et d'une énergie magnétique $W_{\mathrm{M}}$, ces quantités étant rapportées à un atome-gramme.

Energie élastique. - Soit V le volume atomique de la substance au zéro absolu et $d$ la distance de deux atomes proches voisins. Pour une déformation isotrope, le volume devient $\mathrm{V}+\delta \mathrm{V}$ et la distance $d+x$ : l'énergie atomique de déformation se met sous la forme :

$$
\mathrm{W}_{\mathrm{E}}=\mathrm{A} x^{2}+\mathrm{B} x^{3}+\mathrm{C} x^{4}+\ldots
$$

en se bornant aux premiers termes du développement en série et en supposant $x$ petit vis-à-vis de $d$.

Le terme A se calcule au moyen de la compressibilité $\chi_{0}$ au zéro absolu. On sait en effet que pour une très petite déformation, l'énergie emmagasinée par compression s'écrit : $\frac{\mathrm{I}}{2 \psi_{0}}\left(\frac{\delta \mathrm{V}}{\mathrm{V}}\right)^{2} \mathrm{~V}$ et comme dans (2) le terme en $x^{3}$ et les suivants sont négligeables dans ces conditions, on a :

$$
\frac{\mathrm{I}}{2 \gamma_{0}}\left(\frac{\delta \mathrm{V}}{\mathrm{V}}\right)^{2} \mathrm{~V}=\mathrm{A} x^{2}
$$

il faut y joindre la relation évidente :

$$
\frac{x}{d}=\frac{1}{3} \frac{\delta \mathrm{V}}{\mathrm{V}}
$$

et le rapprochement des équations 2 et 3 permet de calculer A. L'extrapolation vers le zéro absolu des valeurs expérimentales de la compressibilité est incertaine, car la variation thermique est grande, aussi les valeurs du tableau I ne peuvent être considérées comme exactes qu'à 10 o/o près. Dans la formule 2, $x$ est exprimé en angströms et W en ergs. 


\section{TABLEAU I}

\begin{tabular}{|c|c|c|c|}
\hline Métal & $\mathrm{Ni}$ & Co & $\mathrm{Fe}$ \\
\hline $\begin{array}{r}x_{0} \times \mathrm{IO}^{12} \\
\mathrm{~A} \times \mathrm{IO}^{-13} \\
-\mathrm{B} \times \mathrm{IO}^{-13}\end{array}$ & $\begin{array}{l}0,50 \\
0,96 \\
2,4\end{array}$ & $\begin{array}{l}0,49 \\
0,99 \\
2,4\end{array}$ & $\begin{array}{l}0,55 \\
0.94 \\
1,94\end{array}$ \\
\hline
\end{tabular}

En principe, B peut se calculer au moyen du coefficient de pression de la compressibilité, mais les valeurs expérimentales de ce coefficient sont beaucoup trop incertaines pour qu'il soit possible d'en tirer parti. Il est préférable de calculer $\mathrm{B}$ au moyen du coefficient de dilatation. Le paragraphe 5 sera consacré à cette question et je discuterai sommairement la précision obtenue dans le paragraphe 12.

Energie magnétique. - On sait que l'on peut ( $\left.{ }^{1}\right)$, à condition de négliger l'effet des fluctuations, remplacer les actions mutuelles entre porteurs de moment par un champ moléculaire $h_{m}$, proportionnel à l'aimantation spontanée $\sigma$;

$$
h_{m}=n \sigma ;
$$

$n$ est le coefficient du champ moléculaire ; l'énergie magnétique s'écrit alors :

$$
\mathrm{W}_{\mathrm{M}}=-\frac{\mathrm{I}}{2} n \sigma^{2} \text {. }
$$

Pour calculer $n$ en fonction de l'énergie d'interaction, on peut évaluer de deux manières différentes l'énergie magnétique correspondant à la saturation absolue, c'est-à-dire à l'orientation parallèle de tous les porteurs.

Soit deu $\mathrm{x}$ atomes portant chacun $q$ spin couplés parallèlement, situés au zéro absolu à la distance $l_{i}$ l'un de l'autre;

(1) Cf. une discussion récente de la question: Weiss. Annales de Phys., 17 (1932), 97. 
lorsque leurs axes magnétiques font entre eux l'angle $\alpha$, l'énergie magnétique s'écrit :

$$
-q^{2} w_{i} \cos \alpha
$$

où $w_{i}$ est fonction de $l_{i}\left({ }^{1}\right)$.

Soit $2 p_{i}$ le nombre des voisins qu'un atome possède à la distance $l_{i}$; quand les spin de tous les atomes sont orientés parallèlement, l'aimantation s'écrit :

$$
\sigma=\mathrm{N} q \mu \cdot(\mu=\text { magnéton de Bohr), }
$$

en désignant par $\mathrm{N}$ le nombre d'Avogadro ; l'énergie magnétique s'écrit, en veillant à ne compter qu'une fois chaque liaison :

$$
\mathrm{W}_{\mathrm{M}}=-\mathrm{N} q_{i}^{2} \sum_{i} w_{i}
$$

le signe somme est étendu à toutes les couches de voisins qui entourent un atome donné. En comparant les relations 6,7 et 8 , on obtient la relation :

$$
n_{0}=\frac{2}{N \mu^{2}} \Sigma_{i} p_{i} w_{i}
$$

dans laquelle j'ai écrit $n_{0}$ pour rappeler qu'il s'agit du coefficient du champ moléculaire au zéro absolu.

Pour simplifier l'écriture, je poserai $\left({ }^{2}\right)$ :

$$
u_{i}=w_{i} \frac{\mathrm{I}}{\mathrm{N} \mu^{2}} .
$$

Il s'agit maintenant de calculer $n$ en fonction de $x$. Pour une déformation isotrope telle que $d$ devienne $d+x$, la dis-

(1) J'ai déjà employé plusieurs fois ce mode de décomposition de l'énergie magnétique. Il conduit à des résultats simples. Voir : NÉEl. Annales de Phys., 17 (1932), 5; J. de Phys., 5 (1934), 104 ; Annales de Phys., 5 (1936), 232.

${ }^{2}$ ) Ce sont ces quantités $u_{l}$ dont j'ai étudié les variations en fonction de la distance des couches magnétiques : NÉ EL. Bull. Soc. Frang. de Phys., no 374 (1935), $9^{3}$ (Dans la dernière colonne du tableau, il faut lire $10^{-2}$ et non pas ${ }^{10^{-25}}$ ) et aussi Annales de Phys., 5 (1936), 232. 
tance $l_{i}$ devient $l_{i}+\frac{x l_{i}}{d}$ et l'énergie d'interaction correspondante s'écrit $w_{i}^{\prime}$ :

$$
w_{i}^{\prime}=w_{i}+\frac{x l_{i}}{d} \frac{\partial w_{t}}{d l_{i}}+\frac{1}{2} \frac{x^{2} l_{i}^{2}}{d^{2}} \frac{\partial^{2} w_{i}}{\partial l_{i}^{2}}+\frac{1}{6} \frac{x^{3} l^{3}}{d^{3}} \frac{\partial^{3} w_{i}}{\partial l_{i}^{3}},
$$

et d'après 8 et 10 le coefficient du champ moléculaire se met sous la forme :

$$
n=n_{0}+n_{1} x+n_{2} x^{3}+n_{3} x^{3},
$$

en posant pour abréger :

$$
\begin{aligned}
& n_{0}=\sum_{i} 2 p_{i} u \\
& n_{1}=\sum_{i} \frac{2 p_{i} l_{i}}{d} \frac{\partial u_{i}}{\partial l_{i}} \\
& n_{2}=\sum_{i} \frac{p_{i} l^{2}}{d^{2}} \frac{\partial^{2} u_{i}}{\partial l_{i}^{2}} \\
& n_{3}=\sum_{i} \frac{p_{i} l_{i}^{3}}{3 a^{3}} \frac{\partial^{3} u_{i}}{\partial l_{i}^{3}}
\end{aligned}
$$

En fonction de $x$, l'énergie magnétique prend la forme :

$$
\mathrm{W}_{\mathrm{M}}=-\frac{\mathrm{I}}{2} \sigma^{2}\left(n_{0}+n_{1} x+n_{2} x^{2}+n_{3} x^{3}\right) .
$$

Cette expression de l'énergie magnétique n'a de sens bien net que si les atomes sont immobiles : par exemple, quand on produit la déformation $x$ par compression au zéro absolu. Au contraire, lorsque la température fait osciller les atomes, on ne peut pas séparer l'énergie thermoélastique ordinaire de la partie de l'énergie magnétique qui est variable avec la température ( $\left.{ }^{1}\right)$.

En principe, pour, calculer les coefficients $n_{0}, n_{1} \ldots$, il faut étendre les signes $\Sigma$ des expressions 13 à toutes les couches successives des atomes qui entourent l'atome central. En pratique, il suffit de se limiter aux deux premières couches pour le système du cube centré et à la première couche seulement pour le système du cube à faces centrées.

(1) Ce point sera précisé dans la $3^{\mathrm{e}}$ partie, $§ 20$. 
Energie totale. - C'est, à une constante près, la somme des énergies élastique et magnétique :

$$
\left.\begin{array}{rl}
\mathrm{W}=\mathrm{W}_{\mathrm{E}}+\mathrm{W}_{\mathrm{M}}=-\frac{\mathrm{I}}{2} n_{0} \sigma^{2}-\frac{\mathrm{I}}{2} n_{1} \sigma^{2} x \\
+\left(\mathrm{A}-\frac{\mathrm{I}}{2} n_{2} \sigma^{2}\right) x^{2}+\left(\mathrm{B}-\frac{\mathrm{I}}{2} n_{3} \sigma^{2}\right) x^{3}
\end{array}\right\}
$$

L'examen de cette expression montre que l'effet de l'aimantation est double :

a) changer la position d'équilibre des atomes au zéro absolu,

b) modifier le coefficient de dilatation.

§ 4. Anomalie de longueur áu zéro absolu. - La nouvelle position d'équilibre $\delta x$ est définie par $\left(\frac{\partial W}{\partial x}\right)=0$, soit :

$$
\delta x=\frac{n_{1} \sigma^{2}}{2\left(2 \mathrm{~A}-n_{2} \sigma^{2}\right)},
$$

au premier ordre près, en négligeant $\left(\mathrm{B}-\frac{1}{2} n_{3} \sigma^{2}\right) \delta x$ devant A $-\frac{1}{2} n_{2} \sigma^{2}$. On peut encore simplifier quand $n_{2} \sigma^{2}$ et $n_{3} \sigma^{2}$ sont respectivement petits devant $A$ et $B$. L'anomalie relative de longueur $\frac{\delta l}{l}$ liée à $\delta x$ par la relation $: \frac{\delta l}{l}=\frac{\delta x}{d}$ s'écrit, au zéro absolu :

$$
\left(\frac{\partial l}{l}\right)_{0}=\frac{n_{1} \sigma^{2}}{4 \mathrm{Ad}}
$$

\$5. Théorie sommaire de la dilatation d'un solide. - Avant d'aller plus loin, il faut exprimer la dilatation d'un solide en fonction des coefficients A et B de l'énergie élastique.

Coefficient d'extension d'une molécule diatomique. Soit une molécule composée de deux atomes. Nous envisagerons uniquement les mouvements de vibration suivant la ligne des centres. Au zéro absolu, la distance d'équilibre est $d$, correspondant à un minimum d'énergie potentielle. 
Lorsque la distance des centres des deux atomes devient $d+x$, l'énergie potentielle de la molécule s'écrit :

$$
w=a x^{2}+b x^{3}+\ldots
$$

A la température T, l'énergie cinétique moyenne et l'énergie potentielle moyenne sont $\left({ }^{1}\right)$ chacune égale à $\frac{k \mathrm{~T}}{\mathrm{a}}$. Comme l'équation ${ }_{17} 7$ renferme un terme en $x^{3}$, les vibrations sont anharmoniques et la distance moyenne des deux atomes devient $d+\delta x$. En négligeant l'effet de la dégénérescence quantique, on trouve :

$$
-\delta x=\frac{3}{4} \frac{b}{a^{2}} k \mathrm{~T} \quad(k=\text { constante de Boltzmann })
$$

et le coefficient d'extension $\alpha$ de la molécule, tout à fait analogue à un coefficient de dilatation, s'écrit :

$$
-\alpha=\frac{3}{4} \frac{b}{a^{2}} \frac{k}{d} .
$$

Chaine linéaire d'atomes. - On peut, de même, prendre une chaîne linéaire d'atomes, supposer que l'énergie entre deux atomes consécutifs est donnée par la formule ${ }_{17}$ et que l'énergie entre deux atomes non consécutifs est négligeable. Le coefficient de dilatation $\alpha$ d'une telle chaine est :

$$
-\alpha=\frac{1}{2} \frac{b}{a^{2}} \frac{k}{d} .
$$

Cette formule est empruntée à Damköhler $\left({ }^{2}\right)$. Elle se déduit par un simple changement de notations de la formule 48 de son mémoire.

Cas général. - D’une manière générale, prenons des atomes disposés suivant un réseau spatial cubique, tel que seules soient à considérer les énergies entre un atome et ses $2 p$ voisins les plus proches à la distance $d$. Lorsque cette

(') Les écarts à l'équipartition, par suite de l'anharmonicité des vibrations, sont négligeables.

( $\left.{ }^{2}\right)$ Ann. der Phys., 24 ( $(\mathrm{g} 935), \mathrm{I}$. 
distance devient $d+x$. l'énergie est donnée par la formule i 7 . Le coefficient de dilatation doit être de la forme :

$$
-\alpha=\mathrm{D} \frac{b}{a^{2}} \frac{k}{d}
$$

$\mathrm{D}$ étant un coefficient numérique qui ne dépend que de la nature géométrique du réseau cristallin. Cette formule peut aussi se justifier par de pures considérations d'équations aux dimensions, en se rappelant que $\mathrm{T}$ ne peut intervenir que sous la forme $k \mathrm{~T}$ qui a les dimensions d'une énergie. Il reste à relier $a$ et $b$ à $\mathrm{A}$ et $\mathrm{B}$. Or chaque liaison de la chaîne, dont l'énergie est représentée par la formule ${ }_{17}$, correspond à un degré de liberté, tandis qu'il y a $3 \mathrm{~N}$ degrés de liberté dans un atome-gramme. Je dois donc écrire :

$$
\mathrm{A}=3 \mathrm{~N} a \quad \text { et } \quad \mathrm{B}=3 \mathrm{~N} b .
$$

La seule difficulté est maintenant de connaître D. Par analogie avec le cas d'atomes en chaînes, j'ai admis $\mathrm{D}=0,5$ : on peut, en somme, s'imaginer un réseau fictif composé de 3 catégories de chaìnes disposées à angle droit les unes par rapport aux autres. D'ailleurs pour formuler la théorie des anomalies de dilatation, il suffit de savoir que D est un nombre pur. Sa valeur numérique n'importe que si l'on se propose de calculer numériquement $n_{2}$ et $n_{3}$.

Dans ce qui précède, on s'est borné à calculer le premier terme du polynome de dilatation défini par:

$$
\frac{\delta l}{l}=\alpha \mathrm{T}+\beta \mathrm{T}^{2}+\ldots
$$

Au prix d'une complexité plus grande de calculs, on aurait pu relier $\beta$ aux coefficients A, B et C de la formule 2 .

§ 6. Expression complète de l'anomalie de longueur d'un solide. - Soit une substance non magnétique dont l'énergie élastique est donnée par la formule 2 . En se plaçant à des températures suffisamment élevées, on pourra négliger la 
dégénérescence du coefficient de dilatation qui se produit à basse température et qui s'interprète identiquement comme la dégénérescence de la chaleur spécifique. Dans ces conditions, la dilatation relative de $0^{\circ}$ à $\mathrm{T} \circ \mathrm{K}$ est donnée par la formule 23 . En prenant $D=0,5$, d'après $2 \mathrm{I}$ et 22 on écrit :

$$
-\alpha=\frac{3}{2} \frac{\mathrm{B}}{\mathrm{A}^{2}} \frac{\mathrm{R}}{d}
$$

$\mathrm{R}$ désigne, comme d'habitude, la constante des gaz parfaits. Supposons maintenant la substance magnétique ; l'expression 2 de l'énergie doit être remplacée par la forme plus complète 15 et le nouveau coefficient de dilatation $\alpha^{\prime}$ est donné par $\left(^{1}\right)$ :

$$
\alpha^{\prime}=\frac{3}{2} \frac{\mathrm{R}}{d} \frac{\mathrm{B}-\frac{\mathrm{I}}{2} n_{3} \sigma^{2}}{\left(\mathrm{~A}-\frac{1}{2} n_{2} \sigma^{2}\right)^{2}} .
$$

Lorsque $n_{2} \sigma^{2}$ et $n_{3} \sigma^{2}$ sont respectivement petits devant A et B, on peut, en tenant compte de 24 , écrire :

$$
\alpha^{\prime}=\alpha\left(1+\frac{n_{2} \sigma^{2}}{\mathrm{~A}}-\frac{n_{3} \sigma^{2}}{2 \mathrm{~B}}\right) .
$$

Il en résulte qu'à la température $T$, la substance magnétique a subi un accroissement de longueur dont l'excès sur l'accroissement correspondant subi par la substance non magnétique est donné par la formule :

$$
\delta l_{\mathrm{T}}=l \sigma^{2}\left(\frac{n_{2}}{\mathrm{~A}}-\frac{n_{3}}{2 \mathrm{~B}}\right) \mathrm{T} .
$$

L'anomalie totale de longueur, c'est-à-dire l'excès de longueur de la substance magnétique, s'obtient en ajoutant à

(1) En réalité, il faudrait d'abord faire un changement de variable pour annuler le terme en $x$ de l'équation $\mathrm{r} 5$, et utiliser les nouveaux coefficients $\mathrm{A}^{\prime}, \mathrm{B}^{\prime}$ du développement ainsi obtenu. Mais l'erreur que l'on commet en simplifiant, comme ci-dessus, est négligeable dans le cas du fer et du nickel. 
l'anomalie au zéro absolu l'anomalie thermique donnée par la formule précédente, d'où :

$$
\frac{\delta l}{l}=\left(\frac{\delta l}{l}\right)_{0}+\left(\frac{\delta l}{l}\right)_{\mathrm{r}}=\sigma^{2}\left[\frac{n_{1}}{4 \mathrm{Ad}}+\alpha \mathrm{T}\left(\frac{n_{2}}{\mathrm{~A}}-\frac{n_{3}}{2 \mathrm{~B}}\right)\right] .
$$

La formule de Chevenard $\left({ }^{1}\right)$, donnée dans le $\$ 2$ (relation I), diffère simplement de la formule 28 par la présence supplémentaire d'un terme en $T^{2}$ qui a été négligé ici pour ne pas alourdir inutilement l'exposé.

En rapprochant l'expression 28 des formules 13 qui définissent les quantités $n_{0}, n_{1}, n_{2}, n_{3}$, on remarque que l'anomalie au zéro absolu permet d'atteindre la dérivée première de l'énergie d'interaction, en fonction de la distance, tandis que la partie thermique de l'anomalie dépend des dérivées suivantes. Un très grand intérêt s'attache ainsi à l étude des anomalies de dilatation, aussi bien pour l'interprétation du phénomène en lui-même que pour les renseiguements qu'il fournit au point de vue du champ moléculaire.

La formule 28 montre qu'il n'y a pas de discontinuité de longueur au point de Curie, on sait d'ailleurs que la perte du ferromagnétisme ne peut être assimilée à un changement d'état. Par contre, il y a discontinuité du coefticient de dilatation; l'anomalie du coefficient de dilatation s'écrit, en effet :

$$
\Delta \alpha=\frac{d}{d t} \frac{\partial l}{l}=\frac{d \sigma^{2}}{d l}\left\lfloor\frac{n_{1}}{4 \mathrm{~A} d}+\alpha \mathrm{T}\left(\frac{n_{2}}{\mathrm{~A}}-\frac{n_{3}}{2 \mathrm{~B}}\right)\right]+\sigma^{2} \alpha\left(\frac{n_{2}}{\mathrm{~A}}-\frac{n_{3}}{2 \mathrm{~B}}\right) .
$$

Au point de Curie, la discontinuité $\Delta \alpha_{\theta}$ du coefficient de dilatation est ainsi :

$$
\Delta \alpha_{\theta}=\left(\frac{d \sigma^{2}}{d \ell}\right)_{\theta}\left[\frac{n_{1}}{4 \mathrm{~A} d}+\alpha \Theta\left(\frac{n_{2}}{\mathrm{~A}}-\frac{n_{3}}{2 \mathrm{~B}}\right)\right] .
$$

\$7. Données expérimentales sur les anomalies de dilatation du nickel. - Expériences de Chevenard. - Après Cheve-

(1) C. R. Ac. d. Sc., 172 (1921), , 655. 
nard, je vais vérifier que les anomalies de longueur d'un ferromagnétique sont exactement représentées par une formule du type:

$$
\frac{\delta l}{l}=\sigma^{2}\left(\lambda+\mu \mathrm{T}+\nu \mathrm{T}^{2}\right),
$$

ce qui constituera une confirmation de la théorie proposée. Je crois utile de reprendre, dans le cas du fer et du nickel, la vérification de Chevenard, car les publications correspondantes sont peu accessibles et peu connues:

Chevenard a étudié, en fonction de la température, la dilatation du fer, du nickel, du chrome et d'un très grand nombre de leurs alliages $\left({ }^{1}\right)$. A partir des très belles courbes données dans son mémoire, j’ai calculé les valeurs du coefficient vrai de dilatation données daus la colonne 4 des tableaux II et III.

Autres données sur le nickel. - La dilatation du nickel a fait l'objet d'études de Colby $\left({ }^{2}\right)$, antérieures à celles de Chevenard, mais faites sur des échantillons de pureté incertaine, aussi ne les reproduirais-je pas. Parmi les travaux ultérieurs, citons ceux de Hidnert $\left({ }^{3}\right)$ sur un échantillon très pur - 99,94\%/o de $\mathrm{Ni}$ - et surtout les expériences de Williams $\left({ }^{4}\right)$ sur un monocristal de nickel contenant $99.900 / 0$ de nickel. Il a fait des mesures précises au voisinage du point de Curie et a trouvé sur la courbe de dilatation un point anguleux situé à $358^{\circ}$, en excellent accord avec le point de Curie du nickel pur - $357^{\circ} 9$ - déterminé par des méthodes magnétiques dans un champ magnétique faible $\left(^{5}\right)$.

(1) Recherches expérimentales sur les alliages de fer de nickel et de chrome. Travaux et mémoires du Bureau International des poids et mesures, 17, Paris, Gauthier-Villars, 1927; voir aussi : P. Weiss, Rapport au Conseil Solvay de 1930.

() Phys. Rev., 30 (1910), 5 о6.

( $\left.{ }^{3}\right)$ Bur. of Stand., 5 (1930), 1305.

(4) Phys. Rev., $46(1934), 1011$.

(5) Nérl. J. de Phys., 6 ( $1935 ;, 27$. 
La figure I rassemble les données des différents expérimentateurs. Elle montre qu'au-dessous du point de Curie, les mesures de Williams sont en excellent accord aussi bien avec celles de Chevenard qu'avec celles de Hidnert.

Au point de Curie, Williams trouve une anomalie d'amplitude plus grande que celle trouvée par Chevenard. Ceci s'explique en partie par le fait que l'échantillon utilisé par Chevenard était moins pur que l'échantillon de Williams, car ce dernier a trouvé que l'amplitude de l'anomalie diminuait de 40 o/0 pour I o/o d'impuretés. Peut-être qu'aussi la discontinuité du coefficient de dilatation au point de Curie est altérée dans une substance à cristallisation confuse et ne possède sa valeur théorique que pour un monocristal. De toutes façons, ce sont les valeurs les plus grandes qui sont les plus sûres.

Au-dessus du point de Curie, dans un intervalle de température d'une cinquantaine de degrés, on remarque que le coefficient de dilatation ne reprend pas immédiatement la marche normale, croissance régulière avec la température, habituelle pour une substance non magnétique. Cette anomalie résiduelle est à rattacher au phénomène des deux points de Curie - point de Curie paramagnétique légèrement différent du point de Curie ferromagnétique - et au phénomène analogue prévu pour la chaleur spécifique $\left.{ }^{(}\right)$et observé par Ahrens $\left({ }^{2}\right)$. Les courbes du pouvoir thermoélectrique en fonction de la température présentent aussi une allure analogue $\left(^{3}\right)$.

A haute température, les expériences de Hidnert s'accor-' dent convenablement avec celles de Chevenard, si on tient compte de la plus grande difficulté des mesures.

D'une manière générale, cette discussion montre que les travaux ultérieurs ont confirmé les résultats de Chevenard

(1) NÉEl. J. de Phys., 5 (1934), ro3.

(') Ann. der Phys., 21 (1934), 169.

(3) Dorfanan, Janus et Kikoin. $Z$. $f$ Phys., 54 (1929). 277. 
obtenus avec son dilatomètre différentiel enregistreur. Comme seules les expériences de Chevenard couvrent un intervalle suffisant de température, comme il a étudié beaucoup d'éléments et d'alliages, j'ai préféré utiliser systématiquement, dans la suite, les résultats de cet auteur.

\$8. Comparaison entre la théorie et l'expérience dans le cas du fer et du nickel. - Détermination expérimentale de l'anomalie de longueur. - La figure I montre qu'au-dessus du point de Curie, le coefficient de dilatation vrai est une fonction linéaire de la température, ce qui facilite l'extrapolatiun vers les basses températures et donne ainsi le coefficient de dilatation de la substance non magnétique. Voici les formules de dilatation qui conviennent le mieux pour le nickel et le fer, quand l'aimantation spontanée est nulle :

$$
\left.\begin{array}{l}
\text { Nickel }: \alpha=12,2 \cdot 10^{-6}+6,2 \cdot 10^{-9} \mathrm{~T} \\
\text { Fer }: \alpha=7,55 \cdot 10^{-6}+7,8 \cdot 10^{-9} \mathrm{~T}
\end{array}\right\}
$$

En retranchant les valeurs données par les formules précédentes, des valeurs réellement observées, on obtient l'anomalie de dilatation.

A la température ordinaire, le coefficient de dilatation est déjà altéré par la dégénérescence quantique. Pour effectuer la correction correspondante, j'ai admis que l'altération relative du coefficient de dilatation était la mème que celle de la chaleur spécifique $\left(^{1}\right)$. Soit $\alpha$ le coefficient de dilatation réel, $\alpha^{\prime}$ le coefficient de dilatation s'il n'y avait pas de dégénérescence, on a :

$$
\alpha=\alpha^{\prime} \Phi\left(\frac{T}{\Theta}\right)
$$

$\Phi(x)$ étant la fonction de Debye $\left({ }^{2}\right)$ et $\Theta$ la température de

(1) On démontre facilement que cette propriété appartient à l'oscillateur biatomique dont il a déjà été question (\$5). Grüneisen (A $/ n$. der Phys., $39(1912), 287)$ a montré que cette relation était approximativement exacte pour un solide.

$\left(^{2}\right)$ inn. der Phys., 39 (19เ2), 789 .

Ann. de Phys., t te série, t. 8 (Octobre ${ }_{19}^{3} 7$ ). 
Debye. J'ai admis ( $\left.{ }^{1}\right): \Theta=37^{\circ}$ pour le nickel et $\Theta=420^{\circ}$ pour le fer. Les valeurs corrigées sont données dans la colonne 5 des tableaux II et III. Dans les colonnes 2 et 3, j'ai indiqué les valeurs du carré $\sigma^{2}$ de l'aimantation spontanée atomique et de sa dérivée ${ }_{d t}$. d'après Weiss et Forrer $\left({ }^{2}\right)$ pour le nickel et Hegg $\left({ }^{3}\right)$ pour le fer.

Dans la colonne 6 , on trouve les anomalies $\Delta \alpha$ du coefficient de dilatation à partir desquelles on a calculé les anomalies relatives de longueur correspondantes (col. 7). En divisant ces dernières valeurs par $\sigma^{2}$, on obtient une expression $\frac{\delta l}{l \sigma^{2}}$ (col. 8) qui doit être une fonction quadratique de la température. J'ai trouvé, en effet, que les expressions :

Nickel $: \frac{\delta l}{l \sigma^{2}}=1,1 \cdot 10^{-11}-7, \mathrm{r} \cdot 10^{-14} \mathrm{~T}+\mathrm{I}, 88 \cdot 10^{-17} \mathrm{~T}^{2}$

Fer $: \frac{\delta l}{l \sigma^{2}}=-1,74 \cdot 10^{-11}+0,95 \cdot 10^{-1+} \mathrm{T}+0,88 \cdot 10^{-17} \mathrm{~T}^{2}(33)$ représentaient bien les valeurs expérimentales comme le montre la comparaison des colonnes 8 et 9 . Enfin dans les colonnes io et 11 , on trouve les valeurs de $\Delta \alpha$ et de $\alpha$ calculées d'après 32 et 33 . J'ai ajouté aux valeurs de $\alpha$ ainsi calculées la correction - négative - de dégénérescence quantique, pour permettre de comparer directement les chiffres de la colonne i i aux données expérimentales brutes.

J'ai tracé, en trait plein, sur les figures 1 et 2 , les courbes calculées donnant le coefficient de dilatation en fonction de la tempér ature. L'accord avec l'expérience est remarquable aussi bien pour le nickel que pour le fer, malgré la complexité des courbes expérimentales notamment dans le cas du fer.

Bref, au point de vue formel, la théorie proposée représente fidèlement les résultats expérimentaux.

(1) Tables de Landolt, $2^{e}$ Supplément.

(2) Annales de Physique, 5 (1926), 153.

(3) Diss., Zurich (1912). 


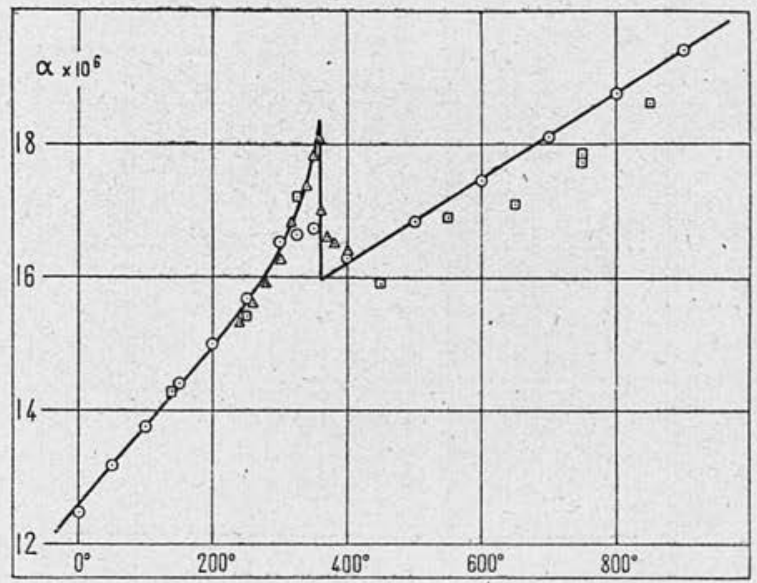

Fig. 1. - Coefficient de dilatation vrai du nickel, en fonction de la température.
$\odot$ Chevenard
$\square$ Hidnert
$\triangle W$ illiams

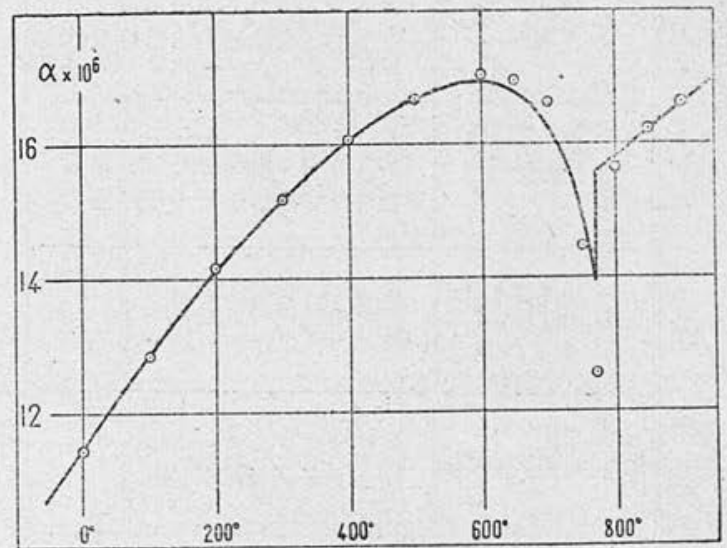

Fig. 2. - Coefficient de dilatation vrai du fer, en fonction de la température. 


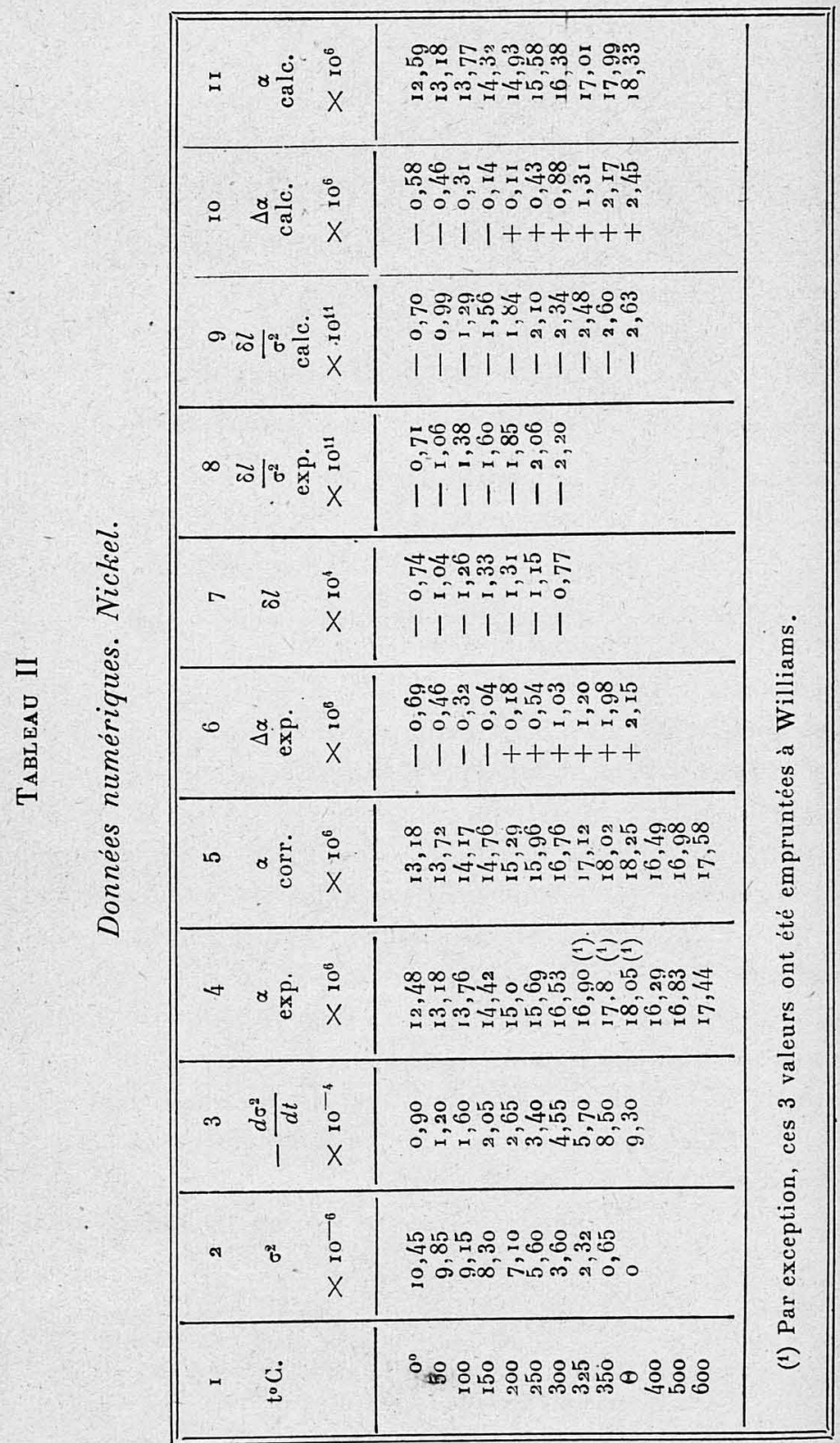




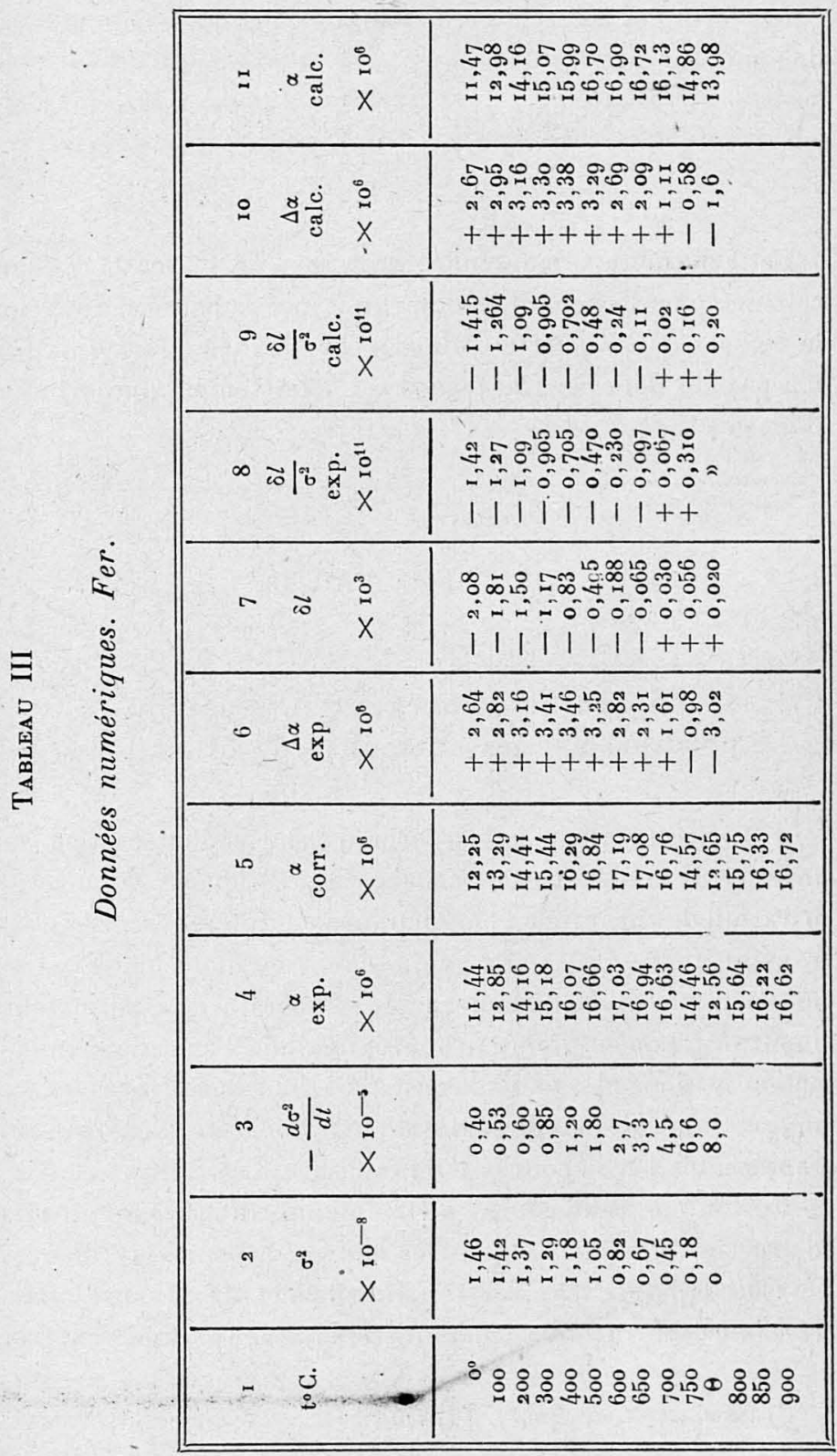


Les formules $3_{2}$ et 33 permettent de calculer les anomalies de longueur au zéro absolu :

$$
\left.\begin{array}{ll}
\text { Nickel }: \frac{\delta l}{l}=+1,25 \times 10^{-4} \\
\text { Fer : } & \frac{\delta l}{l}=-2,66 \times 10^{-3} \cdot
\end{array}\right\}
$$

Cette dernière valeur concorde à peu près avec la valeur calculée par Chevenard, tandis qu'il n'y a pas accord pour le nickel car la correction de dégénérescence quantique qui n'a pas été faite par Ghevenard est relativement importante dans ce cas.

\section{DEUXIÈME PARTIE}

\section{La courbe d'énergie d'interaction précisée par les anomalies de dilatation}

§ 9. Signification de la courbe d'énergie d'interaction en fonction de la distance des couches magnétiques. - La comparaison des propriétés magnétiques de différents corps, ferromagnétiques, paramagnétiques à champ moléculaire, paramagnétiques indépendants de la température, a permis de montrer $\left({ }^{1}\right)$ qu'en première approximation, l'énergie d'interaction ne dépend que de la distance $d-2 r$ entre les couches magnétiques des atomes. En fonction de $d-2 r$, l'énergie est représentée par la courbe $\Gamma$ de la figure 3 .

En somme, cette courbe a été obtenue au moyen d'atomes d'espèces différentes placés, les uns par rapport aux autres, aux distances fixées par les dimensions de la maille du réseau cristallin correspondant. Dans $d \cdot 2 r, d$ représente la

(1) NÉEL. Ann. de Phys., 5 (ז $\left.9^{36}\right), 232$. 
distance des centres des deux atomes et $r$ le rayon de la couche magnétique. Donc, pour obtenir la courbe $\Gamma$, j'ai fait varier $r$, en prenant des atomes différents, tandis que $d$. variait relativement peu, car les distances réticulaires des métaux de la famille du fer sont voisines.

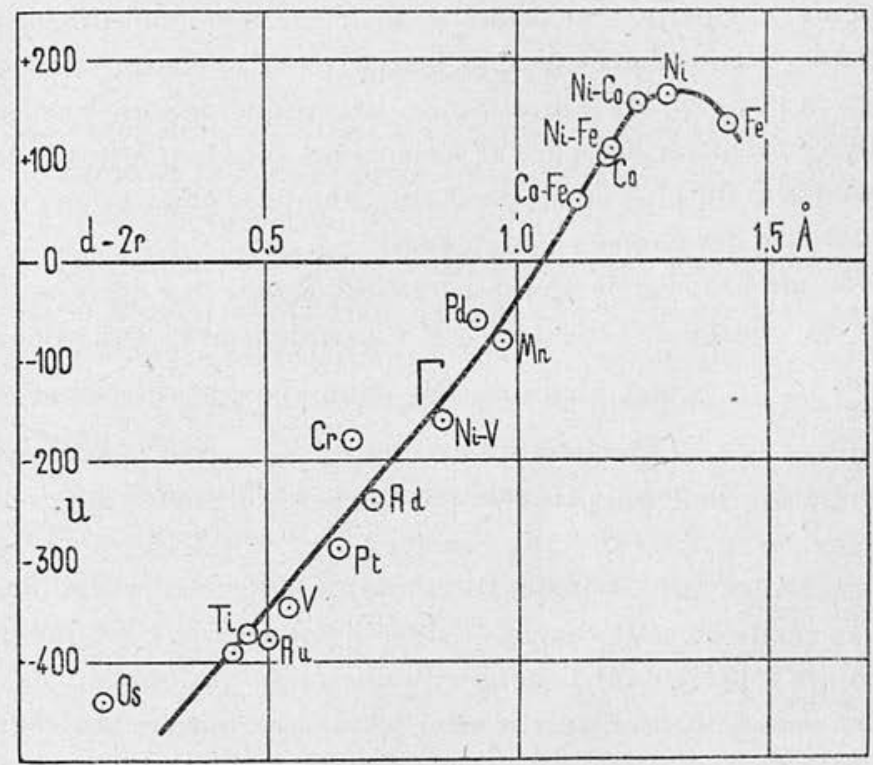

Fig. 3. - Energie d'interaction en fonction de la distance des couches magnétiques.

Réciproquement, soit un atome d'une espèce bien déterminée, un atome de nickel par exemple. Au zéro absolu, la distance entre les couchos magnétiques de deux atomes voisins du réseau est de $\mathrm{r}, 3 \mathrm{o}$ A. Le point correspondant de la courbe $\Gamma$ est le point marqué $\mathrm{Ni}$. Si on modifie artificiellement cette distance, les variations de l'énergie d'interaction seront représentées par la courbe $\Gamma$. En d'autres termes, je décrirai une portion de la courbe $\Gamma$ en laissant $r$ constant et en faisant varier $d$. 
Cependant, on conçoit que pour des atomes d'espèce donnée, l'énergie d'interaction puisse varier suivant une loi - bien déterminée de la distance, sans que l'on puisse ramener à une loi unique les lois relatives à des atomes d'espèces différentes. La théorie des anomalies de dilatation, développée dans la première partie de ce mémoire, se suffit à ellemême: elle permet, en principe, de déterminer, pour un corps donné, le nickel par exemple, les dérivées de l'énergie d'interaction par rapport à la distance. La validité de cette théorie, directement vérifiée par l'expérience est donc tout à fait indèpendan te du plus ou moins d'exactitude des conceptions qui vont être développées maintenant.

Je me propose de montrer que les valeurs des dérivées $\left(^{1}\right)$ de la courbe expérimentale $\Gamma$ s'accordent avec les valeurs $\frac{\partial u}{\partial l}, \frac{\partial^{2} u}{\partial l^{2}} \ldots$ etc., déduites des anomalies de dilatation, ce qui est la conséquence obligatoire de la notion d'existence d'une courbe $\Gamma$ unique, relative à tous les éléments magnétiques.

Ainsi, ce qui est en question maintenant, c'est la significa. tion réelle de cette courbe: s'il n'y a pas accord, c'est que les points représentant l'énergie d'interaction en fonction de la distance sont accidentellement placés sur une courbe régulière. Au contraire, si les deux manières d'obtenir les dérivées fournissentdes résultats concordants, on aura fortement confirmé l'existence de cette courbe et l'étude précise des anomalies de dilatation prendra un très grand intérêt puisqu'on saura en tirer des conclusions générales relatives au champ moléculaire.

Je vais d'abord préciser les relations générales qui lient les dérivées successives de l'énergie à l'anomalie de longueur au zéro absolu et à sa variation thermique. Puis, je passerai à une étude plus détaillée du fer et du nickel.

(1) Dans la théorie des anomalies de dilatation, les dérivées ont été prises par rapport à $d$; elles sont identiques aux dérivées prises par rapport à $d-2 r$, puisque $r$ est constant. 
$\S 10$. Renseignements fournis par l'anomalie de longueur au zéro absolu. - Les formules $\mathrm{r} 3$ et $\mathrm{i} 6$ donnent :

$$
\sum_{i} \frac{2 p_{i} l_{i}}{d} \frac{\partial u_{i}}{\partial l_{i}}=\frac{4 \mathrm{~A} d}{\sigma^{2}}\left(\frac{\partial l}{l}\right)_{0}
$$

Lorsque l'effet de la première couche d'atomes voisins est seul important, cette formule se simplifie et s'écrit :

$$
\frac{\partial u}{\partial l}=\frac{2 \mathrm{Ad}}{\sigma^{2} p}\left(\frac{\partial l}{l}\right)_{0} .
$$

Le signe de l'anomalie de longueur au zéro absolu est aussi le signe de la dérivée première de l'énergie d'interaction par rapport à la distance.

La courbe $\Gamma$ (fig. 3) a un maximum aux environs de $d-2 r={ }_{1}, 3_{1} \AA$. Donc, lorsque la distance des couches magnétiques est inférieure à $\mathrm{I}, 3 \mathrm{I} \AA$, l'anomalie de longueur est positive; au contraire, elle est négative pour les distances supérieures à I, 3 I $\AA$.

J'ai rassemblé dans le tableau IV, pour quelques substances, le signe de l'anomalie de volume ( $\left.{ }^{1}\right)$, la distance $d-2 r$ des couches magnétiques. Les déductions précédentes sont complètement confirmées.

Tableau IV

\begin{tabular}{|c|c|c|c||}
\hline Composé & $\begin{array}{c}\text { Distance } \\
\text { des atomes } \\
\text { magnétiques }\end{array}$ & $\begin{array}{c}\text { Distance } \\
\text { des couches } \\
\text { magnétiques }\end{array}$ & $\begin{array}{c}\text { Signe } \\
\text { de l'anomalie } \\
\text { de longueur }\end{array}$ \\
\cline { 1 - 2 } $\mathrm{Fe}^{3} \mathrm{C}$ & $2,60 \AA$ & $1,16 \AA$ & + \\
$\mathrm{Ni}-\mathrm{Fe}$ & 2,49 & 1,19 & + \\
$\mathrm{Mn}-\mathrm{As}$ & 2,89 & 1,28 & + \\
$\mathrm{Ni}$ & 2,49 & 1,30 & + \\
$\mathrm{Fe}$ & 2,86 & 1,42 & - \\
$\mathrm{Fe}^{2} \mathrm{O}^{3}$ & 2,88 & 1,44 & - \\
$\mathrm{FeS}^{3}$ & 2,89 & 1,45 & - \\
$\mathrm{Fe}^{3} \mathrm{O}^{4}$ & 2,97 & 1,53 & \\
\hline
\end{tabular}

(1) Les études dilatométriques correspondantes ont été faites par Chevenard (loc. cit.) sauf pour AsMn qui a été étudié par Smits, Gerding et Vermast. $Z$. $f$. physik. Chemie; Bodenstein Fetschrift $\left(19^{3} \mathrm{r}\right), 357$. 
Le cas des ferro-nickels est particulièrement intéressant parce que l'on suit à mesure que la proportion de fer augmente, l'augmentation relative de l'anomalie de longueur due au déplacement du point représeńtatif de l'énergie d'interaction vers la gauche de la courbe $\Gamma$, depuis le point $\mathrm{Ni}$ jusqu'au point $\mathrm{Fe}-\mathrm{Ni}$.

Le rayon moyen de la couche magnétique est une donnée indispensable. Ces rayons ont été calculés en utilisant les formules semi-empiriques de Slater $\left({ }^{1}\right)$. En valeur absolue, la précision ainsi obtenue est sans doute médiocre mais on peut espérer que les erreurs commises sont du même ordre et dans le même sens lorsque l'on calcule les rayons moyens de couches magnétiques d'atomes de structures voisines, comme les métaux du groupe du fer, de sorte que l'allure de la courbe $\Gamma$ n'est sans doute pas trop altérée. Par suite, il n'est pas étonnant que l'étude des métaux ait fourni une courbe d'interaction régulière. Mais on peut prévoir que les phénomènes seront plus compliqués pour des composés à caractère polaire. En effet, dans un métal, la couche magnétique a une symétrie sensiblement sphérique tandis que dans un composé comme la magnétite $\mathrm{Fe}^{3} \mathrm{O}^{4}$, à cause des actions électrostatiques, le diamètre de la couche magnétique de l'ion $\mathrm{Fe}^{+++}$doit être plus grand dans la direction des ions $\mathrm{Fe}^{+++}$voisins que dans la direction des ions $\mathrm{O}^{--}$; mais c'est justement le diamètre dans la direction des ions $\mathrm{Fe}^{+++}$ qui nous intéresse et non pas le rayon moyen de la couche.

C'est pourquoi j’ai jugé inutile de discuter quantitativement les propriétés dilatométriques de tels composés: je me suis borné à dresser le tableau IV qui ne saurait fournir que des renseignements qualitatifs.

$\S 11$. Renseignements fournis par l'étude thermique de l'anomalie de longueur. - Examinons la formule 28 qui donne

(') Phys. Rev., 36 ( 1930$), 57$. 
l'expression complète de l'anomalie de longueur. Lorsque la température croìt à partir du zéro absolu, on observe soit un accroissement, soit une diminution de la valeur algébrique de l'anomalie. Comme $\frac{d \sigma^{2}}{d l}$ est nul au zéro absolu (si la loi d'approche à la saturation est en $\mathrm{T}^{3 / 2}$ ), le coefficient du terme en $\mathrm{T}$ de l'anomalie est : $\sigma^{2} \alpha\left(\frac{n_{2}}{\mathrm{~A}}-\frac{n_{3}}{2 \mathrm{~B}}\right)$. Le signe de la variation thermique de l'anomalie est donc le signe de $\frac{n_{2}}{\mathrm{~A}}-\frac{n_{3}}{2 \mathrm{~B}}$.

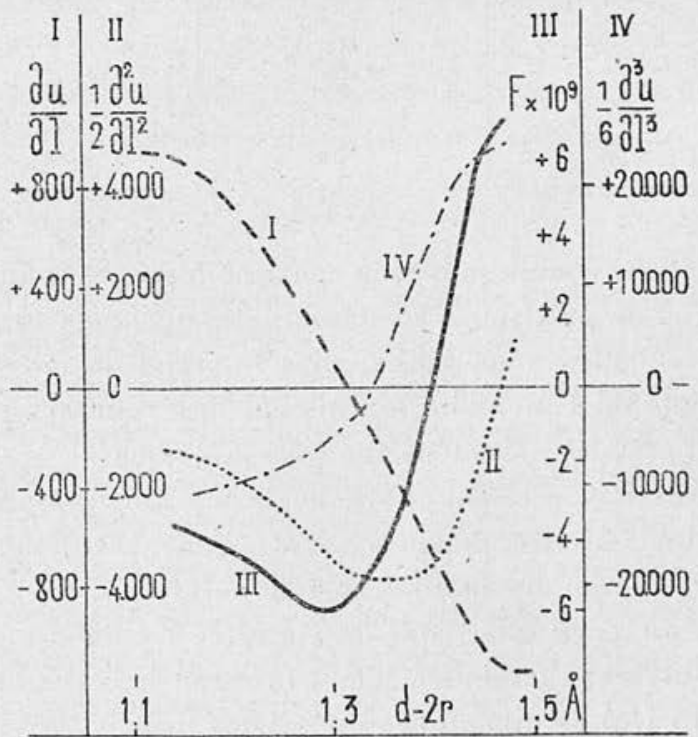

Fig. 4. - Dérivées de l'énergie d’interaction.

J'ai reporté sur la figure 4 , en fonction de $d-2 r$, les valeurs approximatives de $n_{1}, n_{2}, n_{3}$ telles que j'ai pu les déterminer dans les paragraphes suivants. L'échelle des ordonnées est différente pour chacune des courbes. En outre, avec les hypothèses : $p=6$ et $-\mathrm{B}=2 \mathrm{~A}=\mathrm{I}^{+13}$, j'ai tracé la 
courbe $\mathrm{F}=\frac{n_{2}}{\mathrm{~A}}-\frac{n_{3}}{2 \mathrm{~B}}$ en trait plein. On voit que $\mathrm{F}$ d'abord négatif pour les petites valeurs de $d-2 r$, passe par un minimum au voisinage de $d-2 r=1,3 \circ \AA$, puis croît rapidement en s'annulant pour $d-2 r=1,40 \AA$. Les résultats

Tableau V

\begin{tabular}{|c|c|c|}
\hline Composé & $d \cdot 2 r$ & $\begin{array}{c}\text { Signe de la variation } \\
\text { thermique } \\
\text { de l'anomalie }\end{array}$ \\
\hline $\begin{array}{c}\mathrm{Fe}^{3} \mathrm{G} \\
\mathrm{Fe}^{-\mathrm{Ni}} \\
\mathrm{Ni} \\
\mathrm{Fe} \\
\mathrm{Fe}^{3} \mathrm{O}^{4}\end{array}$ & $\begin{array}{l}\text { r, 16 } \AA \\
\text { 1, 19 } \\
\text { r,30 } \\
\text { r, 42 } \\
\text { r, } 53\end{array}$ & $\begin{array}{l}- \\
\overline{-} \\
+ \\
+\end{array}$ \\
\hline
\end{tabular}

sont déjà très compliqués et la courbe F n'est pas connue avec beaucoup de précision. Néanmoins, les quelques substances pour lesquelles nous connaissons le signe de la variation thermique de l'anomalie fournissent des résultats qui s'accordent avec les précisions énoncées ci-dessus.

Les résultats seraient encore beaucoup plus compliqués si l'on voulait décrire, même schématiquement, en fonction de $d-2 r$, l'anomalie de dilatation au point de Curie. Remarquons, en particulier, que le signe de l'anomalie au zéro absolu est tout à fait indépendant du signe de la discontinuité du coefficient de dilatation au point de Curie.

§12. Précision de la méthode. - Les quelques résultats qualitatifs qui viennent d'être obtenus soulignent l'intérêt d'une étude précise de ces phénomènes. Malheureusement, cette étude n'est pas facile.

Pour déduire des anomalies de longueur, l'énergie d'interaction et ses dérivées en fonction de la distance, il faut connaître les quantités A et B, coefficients de la loi d'énergie en 
fonction de la déformation (formule 2). A est directement relié à la compressibilité par les relations 3 et 4 ; mais celle-ci est assez mal connue et dans le cas d'un polycristal, la signification de ce qu'on mesure n'est pas claire : aux fortes pressions employées par Bridgman $\left(^{1}\right)-10000$ atmosphères et davantage - la compression est sans doute accompagnée d'une réorgáanisation de l'édifice polycristallin. En fait, Grüneisen $\left({ }^{2}\right)$ qui a opéré par une méthode indirecte - ondes stationnaires - c'est-à-dire sous des pressions faibles, indique des compressibilités qui diffèrent de plus de ro o/o de celles de-Bridgman. A n'est probablement connu qu'à ı o o près.

Quant à B, la situation est encore plus mauvaise. D'après 24 , on écrit $\mathrm{D}^{\prime}$ étant un coefficient numérique :

$$
\mathrm{B}=\mathrm{N}^{\prime} \mathrm{A}^{2} \alpha d \text {. }
$$

Déjà, la présence du facteur $\mathrm{A}^{2}$ introduit une erreur relative double de celle qui affecte A. $\alpha$ est le coefficient de dilatation au zéro absolu, s'il n'y avait pas de dégénérescence quantique : il est inutile d'insister sur les difficultés d'une détermination précise. $D^{\prime}$ est un coefficient numérique qui ne dépend que de la nature géométrique du réseau. La valeur que j'ai admise n'est pas très certaine. Enfin, la présence de $d$, distance de deux atomes voisins montre que la formule n'est valable que dans le cas où les énergies entre atomes proches voisins interviennent seules. Si, comme dans le fer, il faut faire intervenir la couche suivante, de nouvelles difficultés surgissent de ce fait.

Bref, une très grande prudence s'impose dans l'interprétation des résultats.

(1) Proc. Nat. acad. Amer, 8 (1922), 36r et Proc. Amer, Ac., 58 ( 1923$), 163$.

( $\left.{ }^{2}\right)$ Ann. der Phys., 33 (ig10), 1329. 
§.13. Etude particulière du nickel. - La comparaison des équations 28 et 32 permet d'écrire :

$$
\begin{gathered}
\frac{n_{1}}{4 \mathrm{Ad}}=1, \mathrm{I} \times \mathrm{IO}^{-11} \\
\alpha\left(\frac{n_{2}}{\mathrm{~A}}-\frac{n_{3}}{2 \mathrm{~B}}\right)=-7,1 \times^{-14} .
\end{gathered}
$$

J'adopterai les valeurs numériques suivantes :

$$
\begin{gathered}
\mathrm{A}=0,9^{6} \times \mathrm{IO}^{+13} ; \quad \mathrm{B}=-2,4 \times \mathrm{ro}^{+13} ; \\
d=2,49 \AA ; \quad \alpha=\mathrm{I} 2,2.1 \mathrm{O}^{-6} ; \quad p=6 .
\end{gathered}
$$

Dérivée première de U. - En utilisant la valeur de $n_{1}$ donnée par i 3 , l'équation 38 fournit :

$$
\frac{\partial u}{\partial l}=88 \text {. }
$$

La tangente correspondante s'accorde bien avec ce qui est connu d'autre part de la courbe d'interaction.

Dérivées suivantes de U. - Une relation entre les deux inconnues $\frac{\partial^{2} u}{\partial l^{2}}$ et $\frac{\partial^{3} u}{\partial l^{3}}$ est fournie par 39 .

Une infinité de solutions sont possibles, mais rien ne prouve a priori que parmi elles il s'en trouvera une qui s'accordera avec la courbe $\Gamma$ d'interaction telle que nous la connaissons. Je vais essayer trois systèmes de solutions $a, b$ et $c$ correspondant aux valeurs de $n_{3}$ données ci-dessous :

a) $n_{3}=0$

b) $n_{3}=60000$

c) $n_{3}=120000$

Les trois systèmes de solutions sont caractérisés par les valeurs numériques du tableau VI :

Tableau VI

\begin{tabular}{|c|c|c|c|c||}
\hline \hline Solution & $a$ & $\frac{\partial u}{\partial l}$ & $\frac{1}{2} \frac{\partial^{2} u}{\partial l^{2}}$ & $\frac{1}{6} \frac{\partial^{3} u}{\partial l^{3}}$ \\
\hline$a$ & & 88 & -4650 & 0 \\
\hline$b$ & 88 & -3700 & -5000 \\
\hline$c$ & 167 & -2650 & -10000 \\
\hline
\end{tabular}


A ces trois systèmes de solutions corrrespondent trois courbes tracées sur la figure 5. Les branches de droite de ces courbes sont presque confondues et sont compatibles avec le seul point connu de ce côté de la courbe d'interaction : celui du fer. Ce point est d'ailleurs assez mal connu, car il a été déterminé en supposant nulle ( $\left.{ }^{1}\right)$ l'énergie $u_{1}$ à courte distance $-2,49 \AA-$ ce qui n'est peut-être pas rigoureusement réalisé.

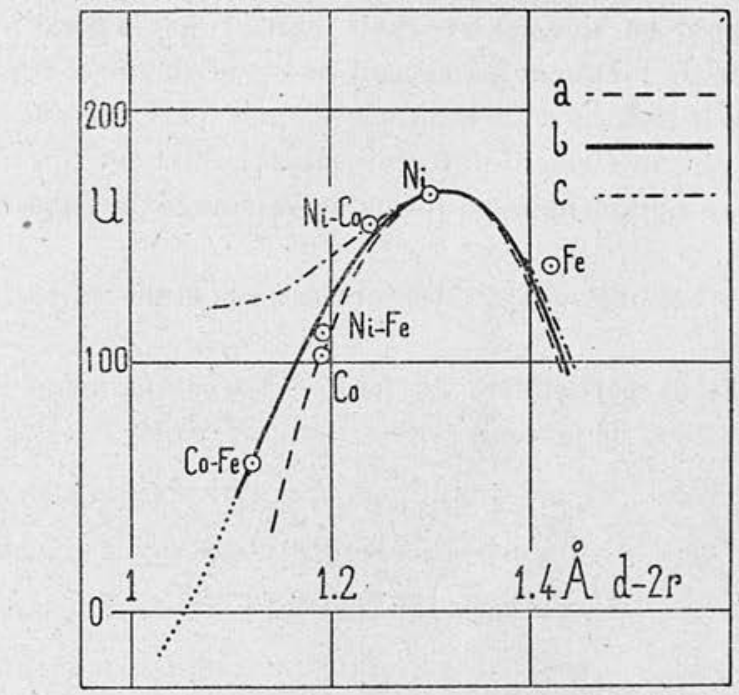

Fig. 5. - Courbes d'énergie d'interaction déduites des anomalies de dilatation du nickel.

L'examen de la branche de gauche des courbes montre que seule convient la solution $b$ ou une solution voisine. Par exemple, pour cette solution, la courbe correspondante traverse l'axe des abscisses au voisinage de $d-2 r=\mathrm{r}, 05 \AA$,

(1) La courbe d'énergie d'interaction étant tracée, on constate qu'elle fournit la valeur $u=0$ pour $d-2 r=1,05 \AA$, ce qui est précisément la distance des couches magnétiques de deux atomes de fer placés à $2,49 \AA$ l'un de l'autre. 
ce qui est tout à fait conforme à ce que nous savons de la courbe $\Gamma$ d'après la figure 3 .

Il serait illusoire de vouloir préciser davantage, car les abscisses des points de la courbe ne sont certainement pas connues à mieux de $0,01 \AA$ près : l'incertitude de la détermination du rayon de la couche magnétique est de cet ordre. De plus, j'ai représenté, dans un intervalle assez grand, la courbe $\Gamma$ par un développement en série, limité au terme en $x^{3}$, d'où des causes d'erreur supplémentaires.

Quoi qu'il en soit, les résultats fournis par l'étude des anomalies de longueur paraissent non seulement compatibles avec la courbe $\Gamma$ déterminée d'autre part suivant des procédés essentiellement différents mais permettent en outre de préciser notablement sa forme au voisinage de l'abscisse du nickel.

Ces conclusions vont être renforcées par l'étude du fer.

$\S$ 14. Etude particulière du fer. - La comparaison des équations 28 et 33 permet, cette fois-ci, d'écrire :

$$
\begin{gathered}
\frac{n_{1}}{4 \mathrm{Ad}}=-1,74 \times 10^{-11} \\
\alpha\left(\frac{n_{2}}{\mathrm{~A}}-\frac{n_{3}}{2 \mathrm{~B}}\right)=0,95 \times 10^{-14} .
\end{gathered}
$$

J'adopterai les valeurs numériques suivantes :
$\mathrm{A}=0,94 \cdot 10^{+13}$;
$\mathrm{B}=-\mathrm{I}, 9+10^{+13}$;
$\alpha=7,55.10^{-6}$.

Sur B, notamment, l'incertitude est extrêmement grande : il n'est peut.être connu qu'à $40 \%$ près.

Deux distances magnétiques entrent en ligne de compte pour le fer, celles qui correspondent aux voisins placés sur les axes ternaires et celles qui correspondent aux voisins placés sur les axes quaternaires. Je les désignerai respectivement par les indices $\mathrm{I}$ et 2 . On a :

$\begin{array}{llll}\text { Distance } 1: & l_{1}=2,49 \AA & u_{1}=0 & p_{1}=4 \\ \text { Distance 2 }: & l_{2}=2,86 \AA & u_{2}=138 & p_{2}=3\end{array}$ 
Pour la distance I, le point représentatif de l'énergie d'interaction est sensiblement sur la longue droite qui représente les énergies négatives d'interaction d'un très grand nombre de métaux à paramagnétisme indépendant de la température ou à point de Curie négatif. La pente de cette droite donne :

$$
\frac{\partial u_{1}}{\partial l}=600
$$

et les dérivées suivantes sont évidemment nulles :

$$
\frac{\partial^{2} u_{1}}{\partial l^{2}}=0 \quad \frac{\partial^{3} u_{1}}{\partial l^{3}}=0 .
$$

D'après les relations $\mathrm{i} 3$ on a :

$$
n_{1}=\frac{p_{1} l_{1}}{d} \frac{\partial u_{1}}{\partial l}+\frac{p_{2} l_{2}}{d} \frac{\partial u_{2}}{\partial l},
$$

et d'après $4 \mathrm{I}$ et 43 et en remplaçant $p_{1}, l_{1}$, etc..., par leur valeur, j’ai :

$$
\frac{\partial u_{2}}{\partial l}=-9^{30}
$$

Même remarque maintenant que pour le nickel : il n'y a que la seule relation 42 pour déterminer $\frac{\partial^{2} u_{2}}{\partial l^{2}}$ et $\frac{\partial^{3} u_{2}}{\partial l^{3}}$. J'essaierai alors trois systèmes de solutions : $a, b$ et $c$ correspondant aux valeurs suivantes de $\frac{1}{6} \frac{\partial^{3} u_{2}}{\partial l^{3}}$ :

$$
\text { 10 000, } 20000 \text { ou } 30000 \text {. }
$$

Dans ces conditions, on remarque que $n_{3} \sigma^{2}$ n'est plus négligeable devant $B$. La formule simplifiée 26 qui représente le coefficient de dilatation de la substance magnétique n'est plus utilisable; on doit revenir à la formule complète 25 : les calculs numériques sont un peu plus longs.

Les trois systèmes de solutions correspondent aux valeurs numériques du tableau VII :

Ann. de Phys., $1^{\text {e }}$ série, t. 8 (Octobre $19^{3} 7$ ). 
Tableau VII

\begin{tabular}{|c|c|c|c|c|}
\hline Solution & $u_{2}$ & $\frac{\partial u_{2}}{\partial l}$ & $\frac{1}{2} \frac{\partial^{2} u_{2}}{\partial l^{2}}$ & $\frac{1}{6} \frac{\partial^{3} u_{2}}{\partial t^{3}}$ \\
\hline $\begin{array}{l}a \\
b \\
c\end{array}$ & $\begin{array}{l}\text { 138 } \\
\text { r38 } \\
\mathrm{r} 38\end{array}$ & $\begin{array}{l}-9^{30} \\
=9^{30} \\
-9^{30}\end{array}$ & $\begin{array}{rl}-1 & 100 \\
-3 & 100 \\
-5 & 000\end{array}$ & $\begin{array}{l}10000 \\
20-000 \\
30000\end{array}$ \\
\hline
\end{tabular}

Les trois courbes correspondantes sont représentées sur la figure 6. Les branches de droite sont assez voisines quelle que soit la solution adoptée. L'énergie d'interaction doit s'annu-

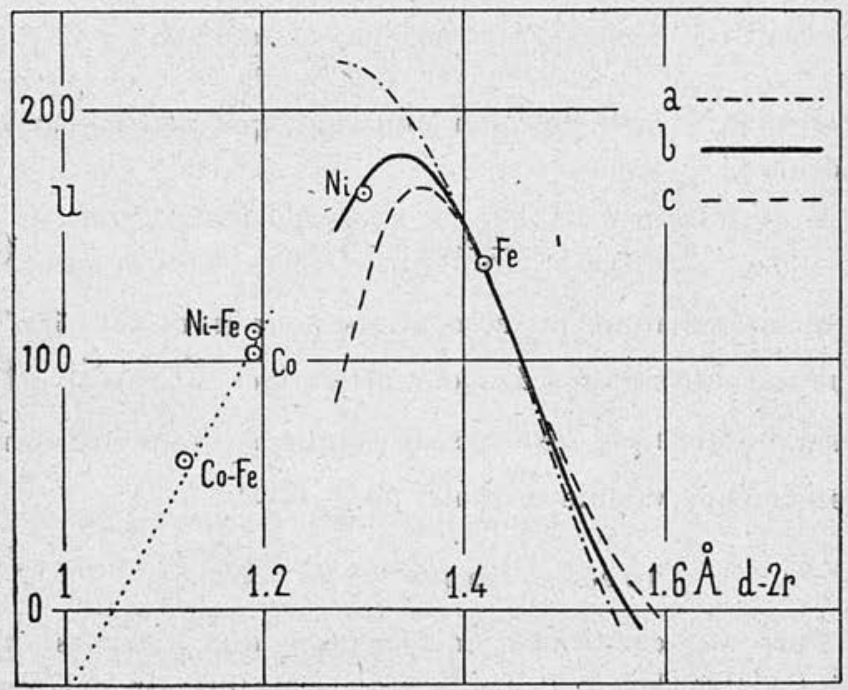

Fig. 6. - Courbes d'énergie d'interaction déduites des anomalies de dilatation du fer.

ler ou tout au moins devenir très petite lorsque $d-{ }_{2} r$ est compris entre $1,55 \AA$ et $1,60 \AA$. La courbe correspondant à la solution $b$ passe au voisinage du point représentatif du nickel. Mais, si on prolongeait cette courbe, on constaterait 
qu'elle ne s'accorderait plus avec les points expérimentaux plus éloignés sur la gauche. Il ne faut pas s'en étonner, un développement en série limité au troisième terme n'est plus valable loin de $x=0$, d'autant plus que la courbe dont il s'agit ici est d'allure très compliquée.

Ainsi, bien quïl ne soit pas possible d'obtenir une très grande précision, l'ensemble des résultats cohérents obtenùs jusqu'ici montre que la représentation schématique que nous nous faisons des phénomènes magnétiques est assez vraisemblable et que les ordres de grandeur des différentes quantités qui interviennent dans la théorie sont bien connus.

\$15. Le point de Curie en fonetion de la pression. - On peut obtenir d'une manière très directe des modifications dans la distance d'interaction en comprimant d'une manière isotrope - pression hydrostatique - une substance ferromagnétique. Le point de Curie doit se déplacer, ce qui permet, en principe, de calculer la dérivée première de l'énergie d'interaction. La théorie précédente permet de calculer com. plètement le déplacement du point de Curie.

Soit $\mathrm{P}$ la pression, $\varkappa_{0}$ la compressibilité ; la variation $\delta l$ de la distance de deux atomes, situés à la distance $l$, lorsque la pression croît de o à $\mathrm{P}$, est :

$$
\delta l=\frac{1}{3} l x_{0} \mathrm{P}
$$

et, toutes choses égales d'ailleurs, le déplacement relatif du point de Curie $\frac{\Delta \theta}{\theta}$ est égal à la variation relative de l'énergie d'interaction, donc :

$$
\frac{\Delta \theta}{\theta}=\frac{\frac{\delta l}{l} \sum_{i} p_{i} l_{i} \frac{\partial u_{i}}{\partial l}}{\sum_{i} p_{i} u_{i}} .
$$

Lorsqu'une seule distance magnétique joue un rôle, la formule 47 se simplifie et s'écrit :

$$
\frac{\Delta \theta}{\theta}=\frac{1}{u} \frac{\partial u}{a l} \delta l .
$$


Les données nécessaires au calcul sont rassemblées dans le tableau VIII. Les compressibilités $x_{0}$ qui y figurent sont les compressibilités au zéro absolu obtenues par extrapolation.

TableaU VIII

\begin{tabular}{|c|c|c|c|c|c|c|c|c|}
\hline Métal & $x_{0} \times 10^{12}$ & $p$ & $u$ & $\frac{\partial u}{\partial l}$ & $l$ & $\theta$ & $\frac{\Delta \theta}{\theta \mathrm{P}} \times 10^{12}$ & $\mathrm{P} \stackrel{\Delta \theta}{=}{ }_{10}{ }^{9}$ \\
\hline $\begin{array}{l}\mathrm{Ni} \\
\mathrm{Co}\end{array}$ & $\begin{array}{l}0,50 \\
0,49\end{array}$ & $\begin{array}{l}6 \\
6\end{array}$ & $\begin{array}{l}167 \\
103\end{array}$ & $\begin{array}{r}88 \\
800\end{array}$ & $\begin{array}{l}2,49 \\
2,49\end{array}$ & $\begin{array}{r}630^{\circ} \\
\times 400^{\circ}\end{array}$ & $\begin{array}{l}0,2 t \\
3,3\end{array}$ & $\begin{array}{l}0^{\circ} 13 \\
4^{\circ} 6\end{array}$ \\
\hline $\mathrm{Fe}$ & 0,55 & $\begin{array}{l}4 \\
3\end{array}$ & $\begin{array}{r}0 \\
138\end{array}$ & $\begin{array}{r}600 \\
-935\end{array}$ & $\begin{array}{l}2,49 \\
2,86\end{array}$ & $1040^{\circ}$ & 0,82 & $-0^{\circ} 79$ \\
\hline
\end{tabular}

Dans la dernière colonne du tableau, on trouve les déplacements du point de Curie calculés pour une augmentation de pression de $10^{9}$ baryes soit 1000 atmosphères environ.

Ces déplacements semblent difficilement mesurables. Ceux du fer et du nickel sont trop petits. Pour le cobalt le point de Clurie est situé à trop haute température. Néanmoins, pour les fer-nickel $\gamma$, les valeurs de $\frac{1}{u} \frac{\partial u}{\partial l}$ doivent dépasser $\gamma$ - valeur du cobalt - quand la proportion de fer alteint 50 o/o. Comme les points de Curie de ces alliages sont facilement accessibles, on pourrait mettre en évidence le déplace. ment prévu qui est de plus de deux degrés, pour 1000 atmosphères.

\section{TROISIÈME PARTIE}

Influence de la variation thermique du champ moléculaire sur les propriétés paramagnétiques.

$\S 16$. Variation thermique de l'énergie d'interaction. L'étude des anomalies de longueur des ferromagnétiques 
confirme l'importance de la variation du champ moléculaire avec la distance des atomes. Donc, l'effet de la température sur le coefficient du champ moléculaire ne doit pas ètre négligeable. Etudions d'abord l'effet sur une liaison isolée entre deux atomes dont la distance est $l_{i}$ au zéro absolu. L'énergie d'interaction, pour une distance $l_{i}+y_{i}$ devient:

$$
u=u_{i}+y_{i} \frac{\partial u_{i}}{\partial l}+\frac{1}{2} y_{i}^{2} \frac{\partial^{2} u_{i}}{\partial l^{2}}+\cdots
$$

$y_{i}$ est une fonction du temps et de la température puisque les atomes oscillent et que leur distance moyenne s'accroit lorsque la température s'élève. On sait d'ailleurs que pour deux atomes voisins l'amplitude des oscillations est grande devant l'écartement moyen provenant de la dilatation. On peut montrer qu'il est légitime de calculer séparément l'effet sur le champ moléculaire de la dilatation et de l'oscillation.

Effet de la dilatation. - En désignant par $\alpha$ le coefficient moyen de dilatation entre $o$ et $\mathrm{T}$, on obtient :

$$
y_{i}=\alpha l_{i} \mathrm{~T}
$$

et la nouvelle valeur de l'énergie s'écrit, en négligeant les termes en $\mathrm{T}^{2}$ :

$$
u=u_{i}+\alpha l_{i} \frac{\partial u_{i}}{\partial l} \mathrm{~T}
$$

Effet de l'oscillation. - Pour simplifier, étudions ce qui se passe à volume constant : les deux atomes considérés oscillent autour de leur position d'équilibre et comme, par hypothèse, il n'y a pas de dilatation la valeur moyenne de $y_{i}$ est nulle; mais la valeur moyenne de $y_{i}^{2}$ n'est pas nulle, désignons-la désormais par : $\bar{y}_{i}^{q}$. L'énergie moyenne d'interaction est donc :

$$
u=u_{i}+\frac{1}{2} \bar{y}^{2} \frac{\partial^{2} u_{i}}{\partial l^{2}} .
$$

Je montrerai dans le paragraphe suivant que $\vec{y}_{i}^{2}$ est propor- 
tionnel à $\mathrm{T}$, de sorte que l'on peut écrire, en combinant les deux équations précédentes :

$$
u_{i}^{\prime}=u_{i}+\left[\alpha l_{i} \frac{\partial u_{i}}{\partial l}+\frac{1}{2 \mathrm{~T}} \bar{y}_{i}^{2} \frac{\partial^{2} u_{i}}{\partial l^{2}}\right] \mathrm{T},
$$

le terme entre crochets étant indépendant de la température.

En multipliant l'équation 53 par $2 p_{i}$ et en ajoutant toutes les équations corres̄pondant à des indices $i$ différents, on a :

$$
\sum_{i} p_{i} u_{i}^{\prime}=\sum_{i} p_{i} u_{i}+\left[2 \alpha \Sigma_{i} l_{i} p_{i} \frac{\partial u_{i}}{\partial l}+\frac{1}{\mathrm{~T}} \sum_{i} p_{i} \bar{y}_{i}^{2} \frac{\partial^{2} u_{i}}{\partial l^{2}}\right] \mathrm{T} .
$$

Or, le premier terme de l'équation précédente n'est autre que $n$, coefficient du champ moléculaire à la température T; mettons d'autre part en évidence $n_{0}$, coefficient du champ moléculaire au zéro absolu, on aura :

$$
n=n_{0}(\mathrm{r}+\lambda \mathrm{T}) \text {, }
$$

en posant :

$$
\lambda n_{0}=2 \alpha \Sigma l_{i} p_{i} \frac{\partial u_{i}}{\partial l}+\frac{1}{T} \Sigma p_{i} \bar{y}_{i}^{2} \frac{\partial^{2} u_{i}}{\partial l^{2}} .
$$

En première approximation, on obtient ainsi pour le coefficient du champ moléculaire une fonction linéaire de la température. Avant d'étudier les conséquences de la formule 55 , je vais dire quelques mots de la détermination de $\bar{y}_{i}^{2}$.

$\$ 17$. Oscillations relatives de deux atomes. - Il s'agit de déterminer le carré moyen $\bar{y}_{i}^{2}$ de l'amplitude de l'oscillation, le long de la ligne qui les joint, de deux atomes situés à la distance $l_{i}$.

Cas d'atomes voisins immédiats. - Si les atomes en question sontimmédiatement voisins, le problème peut être résolu moyennant l'hypothèse suivante : on supposera, qu'au point de vue des énergies élastiques, les actions entre atomes immédiatement voisins sont prépondérantes. Au zéro absolu, 
deux atomes voisins sont en équilibre à la distance $d$, et lorsque la distance des deux atomes devient $d+y$, l'énergie potentielle augmente de:

$$
w=a^{\prime} y^{2}+b^{\prime} y^{3}+\cdots
$$

Il ne faut pas confondre ces coefficients $a^{\prime}, b^{\prime}$, relatifs à une liaison réelle, avec les coefficients $a$ et $b$ du paragraphe 5 qui se rapportaient aux liaisons d'un modèle schématique linéaire.

A partir du zéro absolu, augmentons la température à volume constant. En première approximation, les atomes effectueront des oscillations harmoniques autour de leur position d'équilibre et l'énergie potentielle moyenne d'une liaison se réduit à :

$$
\bar{w}=a^{\prime} y^{2},
$$

et pour les $p \mathrm{~N}$ liaisons d'un atome-gramme, l'énergie petentielle moyenne totale est :

$$
\overline{\mathrm{W}}=p \mathrm{~N} a^{\prime} \bar{y}^{2} .
$$

Mais cette énergie totale est aussi égale à : $\frac{3 R T}{2}$, de sorte que :

$$
\bar{y}^{2}=\frac{3 \mathrm{RT}}{2 p \mathrm{~N} a^{\prime}}
$$

$a^{\prime}$ se calcule immédiatement au moyen de la compressibilité au zéro absolu : en effet, l'énergie potentielle totale emmagasinée dans une déformation statique isotrope, dans laquelle $d$ devient $d+y$, est :

$$
\mathrm{W}^{\prime}=p \mathrm{~N} a^{\prime} y^{2},
$$

mais elle est aussi égale à :

$$
\mathrm{W}^{\prime}=\frac{\mathrm{x}}{2 \mathrm{x}_{0}}\left(\frac{\delta \mathrm{V}}{\mathrm{V}}\right)^{2} \mathrm{~V}=\frac{9}{2 \mathrm{x}_{0}}\left(\frac{y}{d}\right)^{2} \mathrm{~V}
$$


$\mathrm{V}$ étant le volume atomique, car : $\frac{\delta \mathrm{V}}{\mathrm{V}}=3 \frac{y}{d}$ et la comparaison des formules 60 et $6 \mathrm{r}$ fournit :

$$
a^{\prime}=\frac{9 \mathrm{~V}}{2 p \varkappa_{0} d^{2}}
$$

et en reportant cette formule dans l'équation 59 , on obtient finalement :

$$
\bar{y}^{2}=\frac{\mathrm{R} \varkappa_{0} d^{2}}{3 \mathrm{~V}} \mathrm{~T}
$$

L'hypothèse initiale ne joue un rôle que dans la déduction de l'équation 58 qui ne peut être écrite qu'à la condition suivante : les énergies élastiques dues aux couches de voisins autres que la première sont négligeables. Pour un métal à assemblage compact comme le nickel cette condition est assurément réalisée avec une bonne précision et la valeur de $\bar{y}^{2}$ donnée par 62 est correcte.

Grâce à l'artifice qui a été employé, il n'y a eu à faire intervenir ni les amplitudes absolues des oscillations, ni les phases relatives : on a en somme comparé directement les énergies élastiques et magnétiques puisque les mêmes liaisons étaient en jeu dans les deux cas.

Pour un métal comme le fer, à assemblage peu compact, la formule 62 ne donnera qu'une approximation grossière si Y'on donne à $p$ la valeur qui correspond à la première couche de voisins.

Cas d'atomes éloignés. - Par opposition au cas précé. dent, il paraît intéressant de calculer maintenant le carré moyen de l'amplitude relative des oscillations de deux atomes très éloignés. Les phases de leur mouvement seront alors indépendantes et si $\bar{z}^{2}$ est le carré moyen de l'amplitude de l'oscillation d'un atome dans une direction donnée - par rapport à des axes fixes - on aura :

$$
\bar{y}^{2}=2 \bar{z}^{2}
$$


En supposant en première approximation que les atomes oscillent avec une même fréquence moyenne $\nu$ et la même amplitude moyenne $z_{0}$, le mouvement d'un atome sera représenté par l'équation :

$$
z=z_{0} \sin 2 \pi v t
$$

l'énergie cinétique moyenne est $m^{2} \pi \nu^{2} z_{0}^{2}$; elle est égale à $\frac{k \mathrm{~T}}{2}$, donc :

$$
z^{2}=\frac{\varepsilon_{0}^{2}}{2}=\frac{k \mathrm{~T}}{4 \pi^{2} v^{2} m}=\frac{\mathrm{RT}}{4 \pi^{2} v^{2} \mathrm{M}}
$$

M étant la masse atomique.

La fréquence moyenne se détermine en recherchant qu'elle est la température d'Einstein au moyen de laquelle on représente le mieux possible, par une fonction d'Einstein $\left({ }^{1}\right)$, la chaleur spécifique à basse température de l'élément considéré. Soit $\Theta_{E}$ la température caractéristique d'Einstein ainsi déterminée, on a :

$$
k \Theta_{\mathrm{E}}=h \nu .
$$

Dans le tableau suivant, je donne les valeurs de $\theta_{\mathrm{B}}$, les

\begin{tabular}{|c|c|c|c|c|}
\hline & & $\mathrm{Ni}$ & Co & $\mathrm{Fe}$ \\
\hline$\theta_{\mathbf{E}} \cdot . \quad . \quad . \quad . \quad$. & . & $28 I^{\circ}$ & $289^{\circ}$ & $315^{\circ}$ \\
\hline$\frac{\overline{y^{2}}}{\mathrm{~T}} \times 10^{5}:$ atomes voisins & · & $\mathrm{I}, 3 \mathbf{2}$ & I, 28 & $\mathbf{I}, 35$ \\
\hline$\frac{\bar{y}^{2}}{\mathrm{~T}} \times 10^{5}:$ atomes éloignés. & . & 2,08 & 1, $9^{6}$ & $\mathrm{I}, 7^{8}$ \\
\hline e en $\AA . \cdot . \cdot \cdot$ & . & 0,163 & 0,160 & 0,165 \\
\hline
\end{tabular}
valeurs du carré moyen de l'amplitude relative pour des

Tableau IX

(1) On sait que la théorie des chaleurs spécifiques d'Einstein suppose les vibrations monochromatiques. 
atomes voisins et pour des atomes éloignés. On s'aperçoit que les valeurs relatives à des atomes éloignés sont un peu plus grandes que celles qui correspondent à des atomes voisins. En tous cas l'ordre de grandeur des amplitudes relatives et bien établi.

Pour préciser, j'ai calculé à $\mathrm{I} 000^{\circ} \mathrm{K}$., l'écart maximum $e$, par rapport à la distance d'équilibre, de la distance de deux atomes voisins. L'amplitude totale du mouvement dépasse o,3 angström; elle correspond à des variations considérables de l'énergie d'interaction.

$\S 18$. Variation thermique de la susceptibilité. - La susceptibilité d'un paramagnétique pur, dépourvu de champ moléculaire, obéit à la loi de Curie :

$$
\chi=\frac{\mathrm{C}_{0}}{\mathrm{~T}}
$$

en désignant par $\mathrm{C}_{0}$ la constante de Curie. En introduisant un champ moléculaire proportionnel à l'aimantation :

$$
h_{m}=n \sigma,
$$

la susceptibilité est donnée par la loi de Weiss :

$$
\chi=\frac{\mathrm{C}_{0}}{\mathrm{~T}-n \epsilon_{0}} .
$$

Mais d'après l'équation 55 , le coefficient $n$ du champ moléculaire est une fonction linéaire de la température et en explicitant cette dépendance, j’obtiens :

$\chi=\frac{C_{0}}{T-n_{0} C_{0}(\mathrm{I}+\lambda T)}=\frac{\frac{C_{0}}{\mathrm{I}-\lambda n_{0} \mathrm{C}_{0}}}{\mathrm{~T}-\frac{n_{0} \mathrm{C}_{0}}{\mathrm{I}-\lambda n_{0} \mathrm{C}_{0}}}=\frac{\mathrm{C}}{\mathrm{T}-\Theta}$.

La susceptibilité expérimentale de la substance obéit encore à fa Foi de Weiss en ce sens que P̈inverse de la susceptibilité est toujours une fonction linéaire de la température. Posons $\Theta_{0}=n_{0} \mathrm{C}_{0} ; \Theta_{0}$ est le point de Curie que possé- 
derait la substance si elle gardait, lorsque la température varie, le même coefficient de champ moléculaire qu'au zéro absolu. Les relations 67 montrent que la constante de Curie expérimentale $\mathrm{C}$ et le point de Curie expérimental $\Theta$ s'expriment en fonction de $\mathrm{C}_{0}$ et de $\Theta_{0}$ par les relations :

$$
\left\{\begin{array} { l } 
{ \mathrm { C } _ { 0 } = ( 1 - \lambda \Theta _ { 0 } ) \mathrm { C } } \\
{ \Theta _ { 0 } = ( 1 - \lambda \Theta _ { 0 } ) \Theta }
\end{array} \text { ou } \left\{\begin{array}{l}
\mathrm{C}=(1+\lambda \Theta) \mathrm{C}_{0} \\
\Theta=(1+\lambda \Theta) \Theta_{0}
\end{array}\right.\right.
$$

\$19. Champ moléculaire énergétique et champ moléculaire correctif de l'équation d'état. - La formule 67 montre qu'avec une énergie d'interaction qui est une fonction linéaire de la température, la susceptibilité suit formellement la loi de Weiss. Réciproquement, si une substance obéit à la loi de Weiss, l'énergie d'interaction est une. fonction linéaire de la température. Pour démontrer cette proposition, je vais suivre un raisonnement dû à M. Weiss ${ }^{1}{ }^{1}$.

M. Weiss distingue :

Io Un champ moléculaire énergétique défini॰par :

$$
\mathrm{H}_{m}=-\frac{\partial \mathrm{U}}{\partial \sigma}
$$

en désignant par U, l'énergie magnétique de la substance.

La thermodynamique montre que lorsqu'une substance obéit à la loi de Weiss $\left({ }^{2}\right)$ :

$$
\chi=\frac{\mathrm{C}}{\mathrm{T}-\theta},
$$

le champ moléculaire énergétique est proportionnel à l'aimantation et indépendant de la température :

$$
\mathrm{H}_{m}=n_{0} \sigma
$$

(1) J. de Phys., 1 ( $19^{30}$ ), p. 3.

(2) On sait que pour un grand nombre de substances ferromaguétiques cette loi est vérifiée avec précision. Voir par exemple le $\S 7$ du mémoire de M. Weiss : L’hypothèse du cham p moléculaire. Ann. de Physique, 17 (1932), 97. 
et le coefficient de proportionnalité $n_{0}$ est donné par :

$$
n_{0}=\frac{\theta}{\mathrm{C}} \text {. }
$$

$2^{\circ}$ Un champ moléculaire correctif de l'équation d'état $h_{m}$, tel que, en désignant par:

$$
\sigma=f\left(\frac{\mathrm{H}}{\mathrm{T}}\right),
$$

la loi d'aimantation d'un paramagnétique pur, la loi d'aimantation d'un paramagnétique à champ moléculaire soit représenté par l'équation :

$$
\sigma=f\left(\frac{\dot{\mathrm{H}}+h_{m}}{\mathrm{~T}}\right) \text {. }
$$

M. Weiss trouve entre $\mathrm{H}_{m}$ ef $h_{m}$ la relation suivante :

$$
\mathrm{H}_{m}=h_{m}-\mathrm{T} \frac{d h_{m}}{d \mathrm{~T}} \text {. }
$$

Si le champ moléculaire énergétique $\mathrm{H}_{m}$ est indépendant de la température, c'est-à-dire si la substance obéit à la loi de Weiss, l'intégration de l'équation différentielle $7^{5}$ donne $\left({ }^{1}\right)$ :

$$
h_{m}=\mathrm{H}_{m}(\mathrm{I}+\lambda \mathrm{T}),
$$

$\lambda$ étant une constante; le champ moléculaire correctif de l'équation d'état est une fonction linéaire de la température.

M. Weiss a montré que dans le cas du nickel, le coefficient $n_{0}$ du champ moléculaire énergétique était le même à haute

(1) M. Weiss ne considère que la solution: $\mathrm{H}_{m}=h_{m}$. Mais la solution complète montre que la réduction de l'équation expérimentale à la forme de celle des gaz parfaits est possible de diverses manières. Conformément à 67 , en écrivant :

$$
\chi=\frac{\mathrm{C}_{0}}{\mathrm{~T}\left(\mathrm{I}-\lambda n_{0} \mathrm{C}_{0}\right)-n_{0} \mathrm{C}_{0}}
$$

on voit que si l'on part d'une réduction possible, on en a une autre en changeant l'échelle des températures, ce qui était évident. Mais cela ne diminue pas la portée de l'iatroduction de la variabilité de $n$. 
et à basse température $\left({ }^{1}\right)$, ce qui montre la cohérence interne de la théorie du champ moléculaire. La formule 72 montre qu'à haute température, on obtient le coefficient $n_{0}$ du champ moléculaire énergétique et la formule 69 montre que les phénomènes énergétiques, par suite la chaleur spécifique, donneront aussi $\mathrm{H}_{m}$ donc $n_{0}$. Mais de ces résultats on ne peut tirer aucun renseignement sur le coefficient de température, du champ moléculaire correctif de l'équation d'état. Celui-ci est inaccessible directement.

Potter $\left({ }^{2}\right)$ a cru mettre en défaut la constance du coefficient du champ moléculaire énergétique par l'étude du phénomène magnéto-calorique au voisinage du point de Curie. Il a constaté une forte diminution de $n_{0}$, au point de Curie. En réalité cette détermination supposa qu'il est légitime d'utiliser, au voisinage du point de Curie, la notion de champ moléculaire, or j'ai montré $\left({ }^{3}\right)$ que, dans cette région, les fluctuations du champ moléculaire sont telles qu'une valeur moyenne perd toute espèce de signification et les résuitats de Potter loin d'infirmer, comme le pense leur auteur, la théorie du champ moléculaire et de ses fluctuations ne font que souligner leur caractère nécessaire. L'étude des propriétés magnétiques d'une substance au voisinage du point de Curie ne pourra jamais donner d'une manière simple soit le moment, soit le champ moléculaire car c'est une région essentiellement perturbée.

§ 20. Paradoxe apparent d'un champ moléculaire énergétique indépendant de la température. - L'énergie magnéti-

(1) Weiss. Le problème de l'équation d'état des ferromagnétiques. Congrès Solvay (1930), 291 (Gauthier-Villars, Paris 1932); Weiss $J$ de Phys., 1 (1932), 163.

(2) Potrer. Proc. Roy. Soc., 146 (1934), 362.

(3) NÉEL. Ann. de Physique, 17 (1932), 5 ; et J. de Phys., [7], 5 $(1934), 104$. 
que $U$ dont il est question dans le paragraphe précédent a pour expression :

$$
\mathrm{U}=-\frac{\mathrm{I}}{2} n_{0} \sigma^{2}
$$

si la substance obéit à la loi de Weiss; elle est indépendante de la température.

D'autre part, dans le paragraphe 3, équation 14 , j'avais une énergie magnétique $\mathrm{W}_{\mathrm{M}}$ composée d'un premier terme égal à $U$ et de termes supplémentaires dépendant de la température. Cette différence tient uniquement à une question de définition.

Signification de U. - A température constante, les variations de $U$ traduisent les variations de l'énergie interne totale dues aux changements d'aimantation.

Signification de $\mathrm{W}_{\mathrm{M}}$. - A température constante et à volume constant, quand on modifie l'aimantation $\sigma$, l'énergie magnétique $W_{M}$ est modifiée, mais ce que j'ai appelé l'énergie élastique $W_{E}$ - voir paragraphe 3 - est aussi modifié. En effet, les forces qui tendent à ramener les atomes dans leur position d'équilibre sont changées et comme l'énergie cinétique moyenne par degré de liberté reste constante et égale à $\frac{k \mathrm{~T}}{2}$, le carré moyen de l'amplitude change, et par suite l’énergie élastique change aussi.

En d'autres termes, à volume constant, il y a, aussi bien dans l'énergie élastique $W_{E}$ que dans l'énergie magnétique $W_{M}$, un terme qui dépend de la température. Ce terme peut être appelé terme d'oscillation car il provient des oseillations des atomes autour de leur position d'équilibre. Quelle que soit l'aimantation, la somme de ces deux termes d'oscillation est toujours égale à $\frac{3 R T}{2}$, par suite de l'équipartition de l'énergie. Cette somme est égale à l'énergie élastique d'oscillation d'une substance non magnétique.

Tout se passe comme si ténergie magnétique totale était 
indépendante de la température. La thermodynamique donne brutalement le résultat qui est simple mais l'analyse dı mécanisme montre que le processus est complexe. Les variations de l'énergie élastique d'oscillation compensent exactement les variations de l'énergie magnétique d'oscillation. L'étude de la chaleur spécifique ne peut donc donner aucun renseignement sur $W_{M}$ et l'énergie totale de désaimantation qui est facilement accessible expérimentalement est simplement égale à :

$$
\frac{1}{2} n_{0} \sigma^{2}
$$

\$21. Constante de Curie expérimentale et constante de Curie vraie. - Ainsi, le champ moléculaire correctif de l'équation d'état ne coïncide pas avec le champ moléculaire énergétique. Il en résulte que la constante de Curie expérimentale donnée par la loi de Curie-Weiss (70) n'est pas identique à la constante de Curie qu'aurait la substance si an supprimait T'énergie d'interaction sans rien changer d'autre : ce n'est qu'une constante de Curie apparente.

Les relations 68 montrent que la constante de Curie vraie $\mathrm{C}_{0}$ se calcule par la formule :

$$
\dot{c}_{0}=\frac{\mathrm{C}}{\mathrm{I}+\lambda \theta} .
$$

Cette correction. comme je le montrerai dans les paragraphes suivants, est loin d'être négligeable.

Les relations 68 permettent d'écrire, en outre :

$$
n_{0}=\frac{\theta_{0}}{\mathrm{C}_{0}}=\frac{\theta}{\mathrm{C}} \text {. }
$$

On peut détérminer $n_{0}$ indifféremment par le rapport de la constante de Curie apparente au point de Curie ou par le rapport $\frac{\theta_{0}}{\mathrm{C}_{0}}$ qui est plutôt la définition de $n_{0}$. Cette remarque est importante, car elle justifie le mode de détermination de $n_{0}$ que j’ai utilisé dans un mémoire antérieur (1) dans

(1) Ann. de Physique, 5 ( 1936$), 232, \S 13$, éq. 5. 
lequel j'avais supposé que le champ moléculaire était indépendant de la température.

La constante de Curie $\mathrm{C}_{0}$ des paramagnétiques purs s'écrit:

$$
\mathrm{C}_{0}=\frac{\mathrm{r}}{3} \cdot \frac{\mu_{\mathrm{A}}^{2}}{\mathrm{R}} \quad \text { avec }: \quad \mu_{\mathrm{A}}=g \sqrt{j(j+\mathrm{r})} \mu_{\mathrm{B}} .
$$

Dans le cas du fer, du nickel et du cobalt, l'effet gyromagnétique montre que l'on a sensiblement $g=2$, donc le spin seul intervient. Si l'atome porte 1,2 ou 3 spin couplés on a $j=\frac{1}{2}, \frac{2}{2}$ ou $\frac{3}{2}$ et les constantes de Curie correspondantes, atomiques, calculées avec $\mu_{\mathrm{B}}=\mathbf{5 5 5 4}$ sont :

\begin{tabular}{|c|c|c|c||}
\hline \hline$j=$ & $\frac{1}{2}$ & $\frac{2}{2}$ & $\frac{3}{2}$ \\
\hline $\mathrm{C}_{0}$ & $0,37^{2}$ & $0,99^{3}$ & $\mathrm{I}, 862$ \\
\hline
\end{tabular}

Je vais déterminer les constantes de Curie vraies du vickel et du fer et les comparer à ces chiffres.

§ 22. Calcul de la constante de Curie vraie du nickel. - La formule de correction est la formule $78 ; \Theta$ est connu, il s'agit de déterminer $\lambda$. Il faut donc déterminer la dérivée première et la dérivée seconde de l'énergie d'interaction par rapport à la distance.

La dérivée première est donnée sans ambiguïté par l'anomalie de longueur au zéro absolu, on a d'après 40 :

$$
\frac{\partial u}{\partial l}=88
$$

l'angström étant toujours pris comme unilé de longueur. Pour déterminer la dérivée seconde, on peut utiliser les résultats de l'étude la variation thermique de l'anomalie de 
longueur. J'avais obtenu ainsi une valeur voisine de -3700 pour $\frac{1}{2} \frac{\partial^{2} u}{\partial l^{2}}$. Ce résultat présente l'inconvénient d'être basé sur la détermination préalable du coefficient $B$ qui est très mal connu, beaucoup plus mal que A. Cette difficulté peut être évitée de la manière suivaute : on essaiera de faire passer une parabole par les points déjà connus de la courbe d'énergie d'interaction, pris assez voisins de celuidu nickel pour

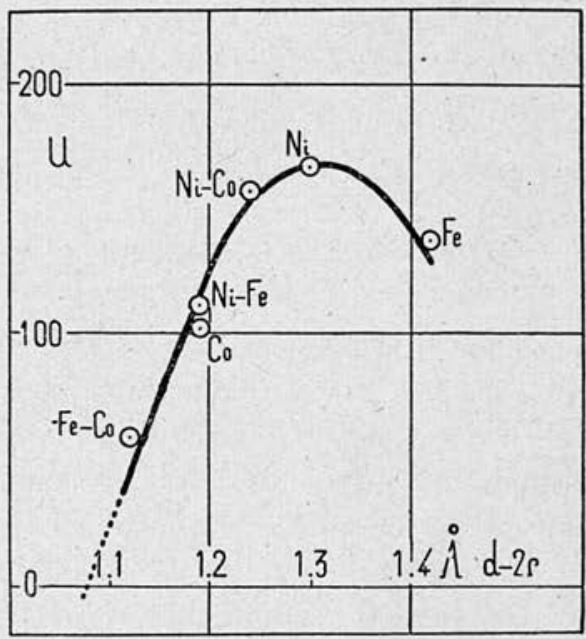

Fig. 7. - Représentation parabolique de la courbe d'énergie d'interaction, au voisinage du point représentatif du nickel.

que les termes du troisième degré puissent être négligés. On utilisera la tangente déterminée plus haut. J'ai trouvé que la parabole suivante :

$$
u=167+88(x-1,30)-3210(x-1,30)^{2},
$$

donnait une bonne représentation comme le montre la figure 7. La dérivée seconde ainsi déterminée s'accorde à Ann. de Phys., t te série, t. 8 (Octobre 1937). 
I 5 o/o près avec la valeur calculée d'après l'anomalie thermique de dilatation. J'admettrai les valeurs suivantes :

$$
\frac{\partial u}{\partial l}=88 \quad . \quad \frac{1}{2} \frac{\partial^{2} u}{\partial l^{2}}=-3210
$$

et, tous calculs faits, on trouve :

$$
\alpha l \frac{\partial u}{\partial l} \theta=2,03 \quad \frac{\mathrm{r}}{2} \bar{y}^{2} \frac{\partial^{2} u}{\partial l^{2}} \theta=-26,6,
$$

d'où :

$$
\lambda \theta=-0,146
$$

La constante de Curie expérimentale du nickel, rapportée à un atome-gramme est $\left({ }^{1}\right): 0,3{ }_{2} 3$; d'où :

$$
\mathrm{C}_{0}=\frac{0.323}{1-0,146}=0,379 \text {. }
$$

Remarquons que si le moment d'un atome de nickel était de 1 magnéton de Bohr, la constante de Curie serait o,372. La différence entre ces deux valeurs n'est pas supérieure aux erreurs possibles. En particulier, la correction peut être affectée d'une erreur de 10 à $15 \%$ o. Je ne pense pas que cette quasi-coïncidence soit due au hasard. Déjà l'étude de l'équation d'état magnétique du nickel $\left({ }^{2}\right)$, plus précisément l'étude de la courbure des isothermes magnétiques au voisinage du point de Curie m'avait permis de conclure que dans l'état paramagnétique, le moment des porteurs était de I magnéton de Bohr à $4 \mathrm{o} / \mathrm{o}$ près. On sait d'ailleurs que la courbe d'aimantation spontanée du nickel s'accorde assez bien avec t'hypothèse de porteurs à 1 magnéton.

D'autre part, j'avais cru, en étudiant les propriétésmagné-

(1) Bloch. Thèse Zurich (1912); Alder. Thèse Zurich (1916); Safranek. Rev. Métall. (fév. 1924); Peschard. Thèse Strasb., $(1,925)$.

(²) NÉвL. J. de Phys, 5 (1934), i 3 . 
tiques des alliages de nickel $\left({ }^{1}\right)$, que le nombre des électrons agissant dans l'état paramagnétique était de o,6 par atome, mais au moment où ce mémoire avait été rédigé, je ne soupconnai pas l'importance des corrections à apporter à la constante de Curie, aussi certaines des conclusions doivent-elles être revisées.

Ainsi, le fait d'attribuer I magnéton de Bohr par atome au nickel ne soulève pas d'objection $\left({ }^{2}\right)$. La configuration électronique des couches incomplètes du nickel serait : $3 d^{9}, 4 \mathrm{~s}$. C'est la couche $3 d$ avec 9 électrons qui joue le rôle d'un porteur paramagnétique à un magnéton de Bohr. L'électron $4 \mathrm{~s}$, comme dans le cuivre dont la configuration est $3 d^{10}, 4 s$, ne joue pas de rôle $\left({ }^{3}\right)$.

\$23. Calcul de la constantelde Curie vraie du cobalt. - On ne possède aucun renseignement sur les anomalies de dilatation du cobalt. Aussi les dérivées de l'énergie d'interaction ne peuvent-elles être déterminées qu'en recherchant le développement en série qui représente au mieux la courbe d'énergie d'interaction au voisinage du point du cobalt. Ce point étant voisin d'un poiut d'inflexion, il est nécessaire d'introduire des termes du troisième degré. Par tâtonnements, on obtient le développement suivant :

$u=106+800(x-1,19)-1400\left(x-1,19^{\times 2}\right)-800(x-1,19)^{3}$.

(1) Ann. de Physique, 5 ( $1936,232$.

(2) Dans l'état ferromagnétique, l'aimantation à saturation est de $0,6 \mu_{\mathrm{B}}$ par atome. A mon avis, ceci ne constitue pas une objection à ce qui a été dit plus haut, mais montre que, contrairement à l'opinion généralement admise, l'aimantation à saturation ne correspond pas à une orientation complète des porteurs. La quatrième partie de ce mémoire est consacrée au développement de cette manière de voir.

$\left({ }^{3}\right)$ D'une façon plus précise, les électrons de cette couche doivent participer à un paramagnétisme constant du type de Pauli (Z.f. Physik., 41 (1927), 97). 
Cette représentation est satisfaisante (fig. 8); en particulier $u$ s'annule pour $x=\mathrm{r}, 06 \AA$, valeur voisine de $x=\mathrm{r}, 05 \AA$ qui a été admise plus haut. J'obtiens ainsi :

$$
\frac{\partial u}{\partial l}=800 \quad \frac{1}{2} \frac{\partial^{2} u}{\partial l^{2}}=-1400 .
$$

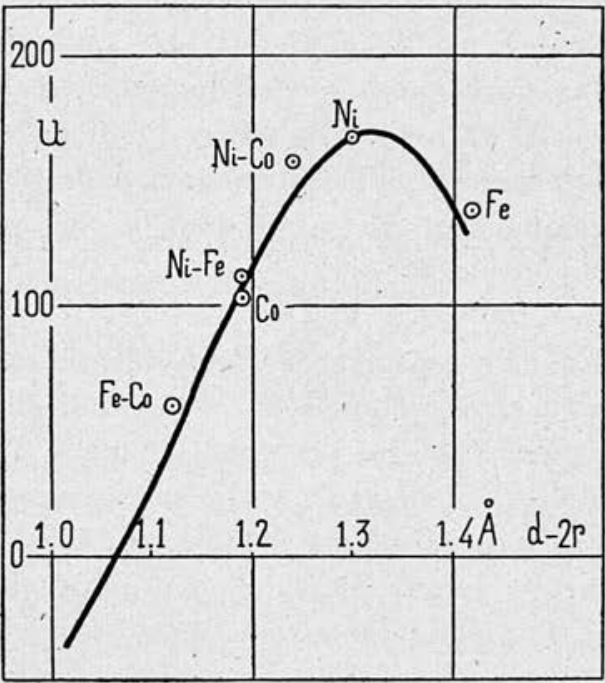

Fig. 8. - Gourbe du troisième degré représentant l'énergie d'interaction au voisinage de l'abscisse du cobalt.

Le coefficient moyen de dilatation, entre $0^{\circ} \mathrm{K}$. et le point de Curie $\left(1400^{\circ} \mathrm{K}\right.$.), n'est pas connu. J'ai admis $14,5 \times 10^{-6}$ valeur moyenne des coefficients moyens de dilatation du nickel et du fer entre $0^{\circ}$ et $1400^{\circ}-$ le nickel et le fer encadrent le cobalt dans le tableau périodique des éléments, ce qui justifie le procédé. Avec ces données numériques, on calcule :

$$
\begin{gathered}
\alpha l \frac{\partial u}{\partial l}-\Theta=40,5, \quad \frac{1}{2} \bar{y}^{2} \frac{\partial^{2} u}{\partial l^{2}} \Theta=-25,2, \\
\lambda . \Theta=0,148 .
\end{gathered}
$$


D'après les expériences de Bloch $\left({ }^{1}\right)$, la constante de Curie atomique du cobalt est $\mathrm{I}, \mathrm{I} 4$; la valeur réelle est :

$$
\mathrm{C}_{0}=\frac{\mathrm{I}, 14}{\mathrm{I}+0,148}=0,99
$$

Il est curieux de remarquer que la correction est de signe contraire à celle du nickel.

La constante de Curie correspondant à 2 spin non compensés par atome est 0,993 . La concordance entre ces deux valeurs est significative : il faut attribuer au cobalt la configuration électronique suivante, pour les couches incomplètes : $3 d^{8}, 4 s$. Etant donné le numéro atomique du cobalt, cette configuration est bien celle à laquelle il fallait logiquement s'attendre connaissant celle du nickel et celle du cuivre.

Ces résultats concordants obtenus pour le nickel et le cobalt se renforcent mutuellement et donnent un appui sérieux à la théorie.

Si l'on considère ces résultats comme acquis, le fer qui possède un électron de moins que le cobalt et deux de moins que le nickel aura la configuration $3 d^{7}, 4 s$, c'est-à-dire 3 magnétons de Bohr par atome.

\$24. Cas du fer. - La constante de Curie atomique, expérimentale du fer est égale $\left({ }^{2}\right)$ à $\mathrm{I}, 23$. Mais la correction indiquée par la théorie précédente n'est pas calculable aveccertitude. En effet, pour les distances entre couches magnétiques supérieures à $\mathrm{r}, 42 \AA$ - distance magnétique du fer - il n'existe pas de points expérimentaux qui permettent de fixer l'allure de la courbe d'interaction et de calculer les termes du développement en série correspondant. Aussi, je prendrai la question en sens inverse; je montrerai que les valeurs des dérivées de l'énergie d'interaction déduites de la correction

(1) Thèse Zurich (I912).

(2) NéEl. C. R. Ac. d. Sc., 193 (1931), г 325. 
à apporter à la constante de Curie expérimentale pour la ramener à la valeur théorique ${ }_{1}, 862$ qui correspond à 3 magnétons de Bohr, jointes aux résultats de l'étude des anomalies de dilatation permettent de calculer une portion de la courbe d'interaction en fonction de la distance qui se raccorde d'une manière remarquable avec ce qui est connu de cette courbe d'autre part.

En effet, on a :

$$
\mathrm{I}+\lambda \theta=\frac{\mathrm{r}, 23}{\mathrm{r}, 86 \mathrm{~g}}=0.660 ; \quad \text { d'où : } \quad \lambda \theta=-0,34
$$

et : $\lambda=3,25 \times \mathrm{ro}^{-4}$. En prenant comme coefficient moyen de dilatation entre $o^{\circ}$ et $\Theta, \alpha=\mathrm{I}_{1}, 6 \times \mathrm{ro}^{-6}$, on en tire, d'après la valeur de la dérivée première (voir $\$ 14$ ) :

$$
\sum_{i} p_{i} u_{i}=414 \quad \sum_{i} p_{i} l \frac{\partial n_{i}}{\partial l_{i}}=2020
$$

d'où :

$$
\sum_{i} \frac{\bar{y}_{i}^{2}}{2} p_{i} \frac{\partial^{2} u i}{\partial t^{2}}=0,114 \quad \text { et : } \quad \frac{1}{2} \frac{\partial^{2} u^{2}}{\partial l^{2}}=-2820 .
$$

Cette valeur ainsi calculée est très voisine. de la valeur -3 100 fournie par l'étude thermique de l'anomalie de dilatation.

Il paraît donc tout à fait justifié d'attribuer 3 magnétons de Bohr au fer.

§ 25. Remarques diverses. - Ce système d'interprétation des constantes de Curie des métaux ferromagnétiques permet de comprendre pourquoi le cobalt et le fer ont des constantes de Curie expérimentales aussi voisines - $\mathrm{I}, \mathrm{I} 4$ et $\mathrm{I}_{2} 3$ alors que leurs moments ferromagnétiques diffèrent relativement bien davantage : c'est que les corrections de variation thermique du champ moléculaire sont de signe contraire et importantes. 
On a découvert depuis peu un quatrième élément ferromagnétique : le gadolinium ( $\left.{ }^{4}\right)$. Sa constante de Curie expérimentale est très voisine de la valeur théorique. Il fallait s'y attendre, car, en admettant un réseau à douze voisins, on trouve pour le gadolinium $u=3,2$ soit une valeur plus de 50 fois plus faible que celle du nickel. D'autre part, la distance entre les couches magnétiques - couches $4 f$ - n'est pas exactement connue, mais elle est certainement très grande : comprise entre 2,5 et $3,0 \AA$. Le point représentatif du gadolinium est donc dans une partie de la courbe d'interaction très rapprochée de l'axe $u=0$, la tangente est petite, la courbure faible, $\lambda$ est ainsi très petit et la correction à apporter à la constante de Curie est négligeable.

Les diamètres de couches magnétiques utilisés dans ce travail ont été calculés $\left({ }^{2}\right)$ en attribuant aux couches magnétiques du nickel, du cobalt et du fer, respectivement 8,7 et 6 électrons. Les paragraphes précédents ont montré qu'il fallait remplacer ces nombres par les suivants : 9,8 et 7 . Rien d'essentiel n'est modifié dans la courbe d'énergie d'interaction; elle doit être simplement décalée, en bloc, d'environ $0,07 \AA$ vers la gauche, ce qui ne présente aucune importance.

J'ai résumé dans le tableau suivant les valeurs les plus probables de l'énergie d'interaction et de ses dérivées, d'après l'ensemble des discussions précédentes.

TABLEAU $\mathrm{X}$

\begin{tabular}{|c|c|c|c|c|c|c|}
\hline Métal & $d$ & $d \cdot 2 r$ & $u$ & $\frac{\partial u}{\partial !}$ & $\frac{1}{2} \frac{\partial^{2} u}{\partial t^{2}}$ & $\frac{1}{6} \frac{\partial^{3} u}{\partial l^{3}}$ \\
\hline $\mathrm{Ni}$ & 2,49 & I, 30 & ${ }_{167}$ & 87 & -3200 & -5000 \\
\hline Co & 2,49 & 1,19 & 103 & 800 & -1400 & -8000 \\
\hline $\mathrm{Fe}_{\mathrm{t}}$ & 2,49 & 1,05 & & 600 & & \\
\hline $\mathrm{Fe}_{2}$ & $2,8 \dot{o}$ & 1,42 & ז 38 & -935 & -3000 & +20000 \\
\hline
\end{tabular}

(1) Urbain, Weiss et Trombe. C. R., 200 (1935), 2132 ; Тrombe. C. R., $201(\mathrm{r} 935)$, г 591.

(2) Ann. de Physique, $5\left(19^{36}\right), 232$. 


\section{QUATRIĖME PARTIE}

Essai d'iṇter prétation du moment ferromagnétique.

§ 26. Le nombre des porteurs orientés est inférieur au nombre total des porteurs. - L'étude des métaux ferromagnétiques, au-dessus du point de Curie, a montré qu'il fallait attribuer I, 2 ou 3 magnétons de Bohr aux atomes de nickel, de cobalt ou de fer, soit sensiblement 5 , io et 15 magnétons de Weiss. Or, on sait que les moments à saturation de ces métaux sont respectivement $3,8,5$ et I I magnétons de Weiss. Le moment orienté, à basse température, est inférieur au moment donné par l'étude du paramagnétisme.

On peut supposer une diminution réelle du nombre des porteurs de moment. Cette hypothèse n'est pas satisfaisante : si, par exemple, le nombre des porteurs variait sous l'influence de la température, il faudrait que cette variation se poursuive dans l'état paramagnétique et la substance ne suivrait pas la loi de Curie-Weiss.

Je penserais plutôt qu'une partie seulement des porteurs est orientée à basse température; le reste serait soustrait au champ moléculaire et ne jouerait par suite aucun rôle dans l'aimantation spontanée.

§ 27. Existence statistique des liaisons magnétiques. - Je s uppose maintenant que les interactions magnétiques entre un atome donné et ses voisins ne sont pas réellement agissantes, chacune d'une manière ininterrompue, mais, qu'au contraire, elles se trouvent réalisées d'une manière essen- 
tiellement discontinue et soumise aux lois du hasard. Etant donné une liaison déterminée, on peut définir la probabilité $\sigma_{i}$ pour que cette liaison se trouve réalisée à un certain instant. Pour un réseau d'un type déterminé, je supposerai que cette probabilité ne dépend que de la distance $l_{i}$ entre les deux atomes entre lesquels s'effectue l'interaction. Elle est indépendante notamment de la valeur des énergies d'interaction et de la nature des atomes entre lesquels s'effectue la liaison. En outre, je supposerai que les probabilités d'occupation des différentes liaisons du réseau sont indépendantes.

Ceci posé, considérons un atome du réseau : à un instant donné deux éventualités peuvent se produire:

a) Aucune des liaisons magnétiques qui le relient au reste du réseau n'est réalisée. Dans ce cas l'atome est entièrement soustrait au champ moléculaire : il n'est pas orienté.

b) Une au moins des liaisons magnétiques est réalisée : l'atome est soumis au champ moléculaire, il est orienté et il participe à-l'aimantation spontanée avec son moment propre.

Si les probabilités d'occupation des différentes liaisons sont connues, un calcul élémentaire donne la probabilité que possède un atome d'être soustrait au champ moléculaire ou au contraire la probabilité qu'il possède d'y participer.

Je pense que le problème de probabilité d'occupation d'une liaison est un problème purement géométrique qui doit être résolu lorsque le réseau cristallin seul est fixé, indépendamment des atomes que l'on mettra aux nœuds du réseau et de leurs énergies mutuelles.

\$28. Calcul des moments atomiques apparents. - Le problème est maintenant de déterminer ces probabilités d'oecupation pour les différents réseaux. Le plus souvent les alliages ferromagnétiques cristallisent : soit dans le système du cube 
à faces centrées, soit dans le système du cube centré. Je me limiterai à ces deux types. Dans ces deux systèmes cubiques, les voisins d'un atome donné peuvent ètre classés sans ambiguïté d'après leur distance à cet atome. Des voisins qui sont placés à la même distance d'un atome central jouent exactement le même rôle vis-à-vis de lui et les liaisons correspondantes sont rigoureusement équivalentes. Je distinguerai les liaisons par des indices, en affectant du même indice les liaisons entre atomes situés à la mème distance, et en affectant des indices les plus faibles celles qui correspondent aux atomes les plus rapprochés.

Le tableau XI donne pour les deux types de réseau envisagés, le nombre des voisins placés sur les différentes " couches" et le rayon des " couches ", en prenant comme unité de longueur la distance des voisins les plus rapprochés.

TABLEaU XI

\begin{tabular}{|c|c|c|c|c|}
\hline & \multicolumn{2}{|c|}{ Cube centré } & \multicolumn{2}{|c|}{ Cube à faces centrées } \\
\hline & Distance & $\begin{array}{l}\text { Nombre } \\
\text { des voisins }\end{array}$ & Distance & $\begin{array}{c}\text { Nombre } \\
\text { des voisins }\end{array}$ \\
\hline I & I & 8 & I & 12 \\
\hline 2 & 1,154 & 6 & 1,414 & 6 \\
\hline 3 & $\mathrm{I}, 632$ & 12 & 1,732 & 24 \\
\hline 4 & $\mathbf{I}, 915$ & 24 & & 12 \\
\hline 5 & 2 & 8 & 2,236 & 24 \\
\hline 6 & 2,30y & 6 & $\therefore$ & . \\
\hline • & $\cdots \cdots$ & - & $\cdots$ & $\cdots$ \\
\hline
\end{tabular}

La probabilité d'occupation d'une liaison doit décroître très vite à mesure que la distance augmente. Le tableau ci-dessus montre que dans le cas du cube à faces centrées, il $\mathrm{y}$ a d'abord $\mathrm{I} 2$ voisins à la distance 1 ; soit $\varpi_{1}$ la probabilité correspondante; on remarque que la couche suivante d'atomes voisins se trouve à la distance 1,414 , c'est-à-dire beaucoup 
plus loin; la probabilité correspondante d'occupation $\varpi_{2}$ doit être heaucoup plus petite que $\omega_{1}$ et en première approximation nous supposerons $\omega_{2} \mathrm{nul}$ ainsi naturellement que les probabilités correspondant aux couches suivantes. On traite ainsi les probabilités comme on a déjà traité les énergies d'interaction : on néglige tout ce qu'il y a au delà de la première couche d'atomes voisins. Bref, tous les systèmes du cube à faces centrées seront caractérisés par une probabilité unique .

Cube centré. - Dans ce cas, le tableau XI montre que les deux premières couches de voisins sont à des distances comparables - $\mathrm{I}_{\text {, }}$,oo et $\mathrm{I}, \mathrm{I} 54$ - il faudra done introduire des probabilités $\varpi_{1}$ et $\varpi_{2}$ relatives à ces deux distances. Pour les couches suivantes on prendra des probabilités égales à o. Le problème est déjà plus compliqué que dans le cas précédent puisqu'il y a deux constantes à déterminer.

\$29. Calcul du moment orienté. Facteur de réduction. - Je traite le cas simple du cube à faces centrées, la généralisation est immédiate. Les seules liaisons qui contribuent au champ moléculaire sont les liaisons entre un atome et ses douze voisoins immédiats. La probabilité de chacune de ces liaisons est ए.

Mais ces douze liaisons ne donnent pas toutes forcément une contribution effective au champ moléculaire ou, pour employer un langage plus concis, ces douze liaisons ne sont pas toutes forcément efficaces. En effet, si l'énergie d'interaction correspondant à une de ces liaisons est nulle, il est évident que tout se passe comme si cette liaison n'existait pas. L'énergie d'interaction peut être nulle: soit quand deux atomes magnétiques sont placés à une distance convenable l'un de l'autre, par exemple deux atomes de fer à $2,49 \AA$, soit qu'il s'agisse d'une liaison entre un atome magnétique et un autre qui ne l'est pas, par exemple un atome de nickel et un atome de cuivre. 
Pour calculer la probabilité que possède un atome d'être soustrait au champ moléculaire, il faudra calculer la probabilité pour qu'aucune des liaisons $\epsilon$ fficaces ne soit réalisée et non pas la probabilité pour qu'aucune des 12 liaisons ne soit réalisée. Soit $n$ le nombre des liaisons ou voisins efficaces; $n$ est égal ou inférieur à $\mathbf{2} 2$.

La probabilité pour qu'une liaison efficace soit occupée étant égale à $\varpi$, la probabilité pour qu'aucune des $n$ liaisons efficaces ne soit occupée est : $(\mathrm{I}-\varpi)^{n}$. Dans ce cas, l'atome n'est pas orienté, son moment n'entre pas en ligne de compte dans l'aimantation à saturation. La probabilité pour qu'une au moins des liaisons efficaces soit occupée est $\mathrm{I}-(\mathrm{I}-\varpi)^{n}$; c'est aussi la probabilité pour qu'un atome soit orienté; si le moment magnétique d'un atome est égal à $\mu_{\mathrm{B}}$, le moment moyen par atome, correspondant à l'aimantation à saturation sera :

$$
\mu=\left[\mathrm{r}-(\mathrm{r}-\omega)^{n}\right] \mu_{\mathrm{B}}=f \mu_{\mathrm{B}},
$$

en posant $f=\left[1-(1-\varpi)^{n}\right]$ et en appelant $f$ le facteur de réduction du moment.

Le raisonnement précédent est valable pour des atomes ,portant un seul électron non compensé, car la liaison magnétique correspondant à deux atomes voisins est simple. Les choses sont un peu plus compliquées lorsque chaque atome porte plusieurs électrons non compensés. Supposons, par exemple, qu'il y ait 3 électrons non compensés par atome. Chaque liaison doit être considérée comme triple puisqu'il peut y avoir 3 liaisons réalisées en même temps. Un atome possède donc $3 n$ liaisons magnétiques efficaces. La probabilité d'occupation reste la même, car si le nombre des liaisons a été triplé, on a triplé également le nombre des électrons qui les réalisent.

Pour avoir le moment moyen par atome, il suffira donc de remplacer dans la formule $83, \mu_{\mathrm{B}}$ par $3 \mu_{\mathrm{B}}$ et $n$ par $3 n$ :

$$
\mu=3\left[1-(1-\varpi)^{3 n}\right] \mu_{\mathrm{B}} .
$$


D'une manière générale, le facteur de réduction, $f$, par lequel il faut multiplier le moment théorique total pour avoir le moment orienté, c'ẹst-à-dire le moment expérimental, s'écrit :

$$
f=\mathrm{I}-(\mathrm{1}-\varpi)^{n q} ;
$$

en désignant par $n q$ le produit du nombre $n$ des voisins efficaces par le nombre $q$ des spin non compensés par atome.

Cette formule n'est applicable que dans le réseau du cube à faces centrées; pour le cube centré, il faut faire intervenir deux probabilités, néanmoins, à titre de première approximation on pourra utiliser dans ce cas la formule 85 avec la constante $\varpi$ correspondant au cube à faces centrées.

$\S 30$. Discussion des moments calculés dans les réseaux cubiques à faces centrées (réseaux $\gamma$ ). - La seule constante arbitraire que renferme la formule 85 est $\varpi$. En effet, pour le nickel, le cobalt et le fer le nombre des spin non compensés par atome est connu : il est respectivement de 1,2 et 3 pour ces différents métaux. Le nombre $n$ des voisins efficaces s'obtient par une discussion facile en utilisant les constantes géométriques du réseau et les énergies d'interaction antérieurement déterminées.

Je déterminerai б d'après le moment expérimental du nickel ; dans ce cas, en effet, on a : $f=0,6 ; n=12 ; q=1$. Avec ces donuées, on obtient :

$$
\varpi=0,07^{333 .} .
$$

Ceci admis, il est possible de calculer a priori le moment de tous les atomes ferromagnétiques, selon la nature des atomes voisins, sans introduire de nouvelles constantes arbi- 
traires. Le tableau XII donne quelques résultats. La notation $\mathrm{Fe}_{6}$ indique un atome de fer à 6 voisins. Les moments ont été uniformément évalués en magnétons de Weiss.

Tableau XII

Base : $\mathrm{Ni}_{12}=3$.

\begin{tabular}{|c|c|c|c|c|c|}
\hline Réseau & Atome & $q$ & $n q$ & $\begin{array}{l}\text { Moment } \\
\text { calculé }\end{array}$ & $\begin{array}{l}\text { Moment } \\
\text { observé }\end{array}$ \\
\hline $\begin{array}{l}\text { Cube à faces } \\
\text { centrées }\end{array}$ & $\begin{array}{l}\mathrm{Ni}_{11} \\
\mathrm{Ni}_{12} \\
\mathrm{Co}_{12} \\
\mathrm{Fe}_{12}\end{array}$ & $\begin{array}{l}\mathbf{I} \\
\mathbf{I} \\
2 \\
3\end{array}$ & $\begin{array}{l}\text { II } \\
\text { 12 } \\
24 \\
36\end{array}$ & $\begin{array}{l}2,84 \\
3 \text { (base) } \\
8,40 \\
14,04\end{array}$ & $\begin{array}{r}2,83 \\
3,00 \\
8,49 \\
14,00\end{array}$ \\
\hline Cube centré & $\begin{array}{l}\mathrm{Ni}_{14} \\
\mathrm{Co}_{14} \\
\mathrm{Fe}_{6} \\
\mathrm{Fe}_{7} \\
\mathrm{Fe}_{8} \\
\mathrm{Fe}_{10} \\
\mathrm{Fe}_{14}\end{array}$ & $\begin{array}{l}1 \\
2 \\
3 \\
3 \\
3 \\
3 \\
3\end{array}$ & $\begin{array}{l}14 \\
28 \\
18 \\
21 \\
24 \\
30 \\
42\end{array}$ & $\begin{array}{r}3,28 \\
8,82 \\
11,19 \\
11,97 \\
12,59 \\
13,47 \\
14,39\end{array}$ & $\begin{array}{r}9,1 \\
11,0 \\
14,6\end{array}$ \\
\hline
\end{tabular}

Je vais discuter rapidement les résultats obtenus.

Fer à douze voisins. - C'est l'état du fer en solution solide dans le réseau du nickel. Peschard (1) a établi que le moment atomique variait linéairement en fonction du titre depuis le nickel pur jusqu'à 3o o/o Fe atomique. Par extrapolation, on obtient avec précision le moment du fer à I 2 voisins : I 4,00 $\pm 0,05$ magnétons. $L$ 'accord avec la valeur calculée est excellent.

Cobalt à douze voisins. - A la température ordinaire, le cobalt cristallise dans le réseau hexagonal compact $\left({ }^{2}\right)$. Les douze voisins les plus proches sont géométriquement placés de deux manières différentes, mais sont exactement à la même distance, 2,5r4

(1) Thèse Strasbourg, ${ }_{1925}$ et Revue de Métallurgie (1925).

$\left({ }^{2}\right)$ Ewald u. Hbrmann. Strülturbericht, Leipzig $\left({ }^{1} 9^{31}\right)$. 
paraît légitime d'appliquer les mêmes formules que pour le système du cube à faces centrées. Le calcul donne 8,40 magnétons, l'expérience (') 8,49 magnétons : l'accord est encore très bon.

Solutions solides de nickel et de cuivre. - Si l'on attribue au nickel la configuration $3 d^{9} 4 s$, le cuivre qui possède I électron de plus aura la configuration $3 d^{10} 4 s$ soit une couche $3 d$ complète et un moment égal à zéro. La courbe des moments atomiques des solutions solides de nickel et de cuivre doit donc être une droite joignant le moment 3 du nickel au moment zéro pour le cuivre.

Ce raisonnement simple n'est pas tout à fait exact. En effet, quand on remṕlace un atome de nickel par un atome de cuivre, le moment total diminue : d'abord de 3 magnétons par la disparition de l'atome de nickel, mais, en outre, les douze atomes de nickel qui entourent l'atome de cuivre que l'on vient d'introduire possèdent désormais 11 voisins nickel et I voisin cuivre, c'est-à-dire seulement I I voisins efficaces. Or, le moment de $\mathrm{Ni}_{11}$ est 2,837 soit o, 163 magnéton de moins que $\mathrm{Ni}_{12}$. Par atome de cuivre introduit, la perte de moment supplémentaire est donc $12 \times 0,163$ $=\mathrm{r}, 96$ magnéton. Du còté du cuivre, la droite des moments doit donc ainsi s'extrapoler vers - 1,96 magnéton. L'expérience $\left(^{2}\right)$ donne $-2,0$. L'accord ne pourrait pas être meilleur.

Cette interprétation des propriétés magnétiques des nickelcuivre me parait intéressante, car, jusqu'ici, ce problème n'avait reçu que des solutions peu satisfaisantes.

Le gadolinium. - On a trouvé $\left({ }^{3}\right)$ pour le gadolinium un moment à saturation de 35,4 magnétons égal, aux erreurs

(1) Weiss et Forren. Ann. de Phys., 5 ( $\left.19^{26}\right)$, i 53.

( ${ }^{2}$ Alder. Thèse Zurich $(1916)$. Voir aus i : Forrer. « Les principes de discussion des moments atomiques, dans les ferromagnétiques ». J. de Phys., 1 (' $9^{30}$ ', 325.

$\left({ }^{3}\right)$ G. Urbain, P. Weiss et F. Trombe. $G$ R., 200 ( 1935$), 2$ ז32. 
d'expériences près, au moment théorique $-34,8$ magnétons - En prenant le nombre des voisins efficaces égal à $\mathbf{1 2}_{2}$, le produit $n q$ est égal à 84 et le calcul montre que le facteur de réduction, $f$, est égal à $0,99^{8}$. A 2 o/oo près, le moment orienté est égal au moment total.

$\S 31$. Discussion des moments calculés dans les réseaux du cube centré (réseaux $\alpha$ ). - Il ne faut pas s'attendre à avoir ici des concordances aussi bonnes que dans le paragraphe précédent, puisque j'ai remplacé les deux probabilités relatives à ce type de réseau par la probabilité unique du réseau $\gamma$.

$\mathrm{Fer}$ a. - Le fer ne possède que 6 voisins efficaces à la distance de 2,86 angströms. Les 8 voisins des axes ternaires sont à une distance d'interaction nulle. Le calcul donne I I , 19 magnétons, l'expérience II , oo.

Ferro-cobalts $\alpha$. - Ils forment une solution solide dans le réseau $\alpha$ depuis le fer jusqu'à $78 \%$ de cobalt. Les moments magnétiques atomiques ont été mesurés avec précision par Weiss et Forrer ${ }^{(1)}$.

On peut supposer, comme cela se produit souvent dans le réseau $\alpha\left({ }^{2}\right)$, l'existence de surstructures à : $\frac{1}{16}, \frac{1}{8}, \frac{1}{4}$ et $\frac{1}{2}$ de cobalt. Pour chacune de ces surstructures, on peut déterminer le nombre des liaisons efficaces de chaque atome $\left(^{3}\right)$ et calculer le moment atomique moyen. En supposant, entre deux structures consé:utives, une variation linéaire du moment, on obtient les lignes brisées tracées en pointillé sur la figure 9 .

(1) Annales de Physique, 12 (1929), 279.

(2) Voir au sujet de la détermination magnétique de telles surstractures, la thèse de Fallot (Strasbourg, 1935). L'hypothèse faite ici n'est pas essentielle. Si on suppose au contraire une répartition statistique des atomes de cobalt et de fer, les courbes calculées ne sont pas modifiées de manière profonde.

(3) Pour le détail du calcul, se reporter au $\$ 33$. 
Quand la proportion de cobalt est supérieure à $50 \%$, tous les atomes ont 14 voisins efficaces : l'alliage n'est plus qu'un mélange de $\mathrm{Fe}_{14}$ et de $\mathrm{Co}_{14}$. Le calcul donne respectivement pour ces deux moments 14,39 et 8,82 tandis que l'expérience doune 14,6 et $9, \mathrm{r}$.

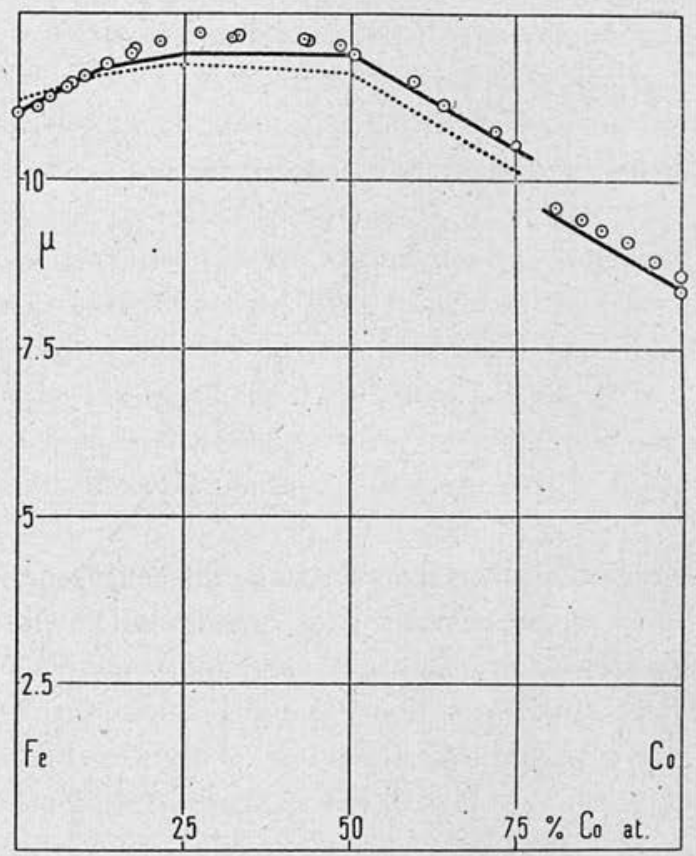

Fig. 9. - Moments atomiques à saturation des ferro.cobalts.

Ferro-nickels $\alpha$. - Ils forment une solution solide dans le réseau $\alpha$ depuis le fer pur jusqu’à 33 o/o de nickel. Les moments magnétiques atomiques ont été mesurés par Peschard ( $\left.{ }^{1}\right)$ Le calcul de la courbe des moments atomiques est identique au calcul de la courbe des ferro-cobalts. Il a donné la ligne brisée tracée en pointillé sur la figure io.

(1) These Strasbourg, 1925.

Ann. de Phys., $11^{\text {e }}$ série, t. 8 (Octobre ${ }_{1937}$ ). 
Certes, les tracés calculés ne représentent pas avec précision les courbes expérimentales, mais comme ils ont été obtenus en supposant connu le seal moment du nicket le résultat est cependant $\cdot$ satisfaisant.

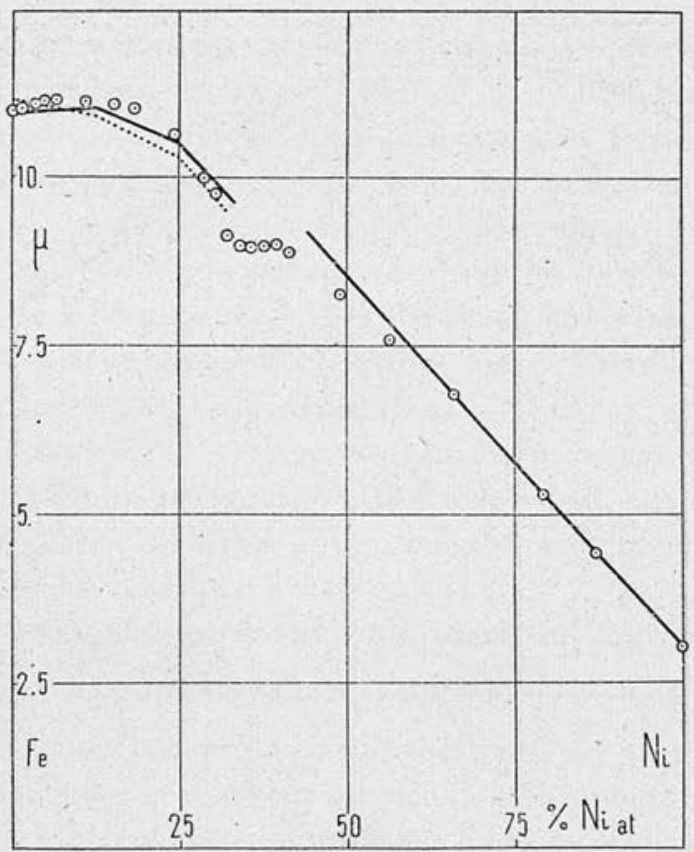

Fig. 10. - Moments atomiques à saturation des ferro-nickels.

Deuxième approximation pour le réseau $\alpha$. - Pour perfectionner le calcul du moment, on peut introduire les deux probabilités $\varpi_{1}$ et $\varpi_{2}$ relatives aux deux catégories de liaisons du réseau $\alpha$. Ces deux inconnues se calculeront au moyen de deux moments considérés comme connus par exemple : $\mathrm{Fe}_{6}={ }_{11}, 00$ et $\mathrm{Fe}_{14}=14,60$. On trouve ainsi : $\varpi_{1}=0,09148$ et $\varpi_{2}=0,0708$ o. 
Ces valeurs permettent de calculer les moments suivants : $\mathrm{Ni}_{14}=3,50 ; \mathrm{Co}_{14}=9,11 ; \mathrm{Fe}_{7}={ }_{12}, 00 ; \mathrm{Fe}_{8}=12,75 ;$ $\mathrm{Fe}_{10}=13,7$.

Le moment 9,11 pour $\mathrm{Co}_{14}$ est exactement celui que fournit l'expérience (ferro cobalts de 50 o/o à $750 / 0$ de cobalt). De plus, à partir des moments calculés ci-dessus, on déduit qu'au voisinage du fer, dans les ferro-cohalts, le début de la courbe des moments atomiques s'extrapole, pour le cobalt pur, vers 17,11. L'expérience donne presque exactement la même valeur : ${ }_{1} 7,0$ d'après Forrer $\left(^{1}\right)$.

Avec ces nouvelles valeurs, on calcule que le moment atomique varie en fonction du titre suivant les courbes tracées en traits pleins sur les figures 9 et 10 . L'accord est bien meilleur que tout à l'heure, sans être parfait. Il faut noter cependant que la différence entre les moments calculés et les moments observés n'atteint pas $3 \%$.

\$32. Rapprochements entre les considérations précédentes et la théorie de Heisenberg. - Les concordances numériques mises en évidence dans les deux paragraphes précédents sont assez frappantes. Dans plusieurs cas, les moments calculés coïncident avec les moments observés avec une précision atteignant le $\mathrm{I} / 200^{\mathrm{e}}$. Je n'ai pas cependant l'impression que ces faits constituent une vérification de la théorie assez audacieuse que j'ai avancée. J'ai plutôt le sentiment que la formule 85 , qui donne le facteur de réduction du moment en fonction du produit $n q$, est exacte mais que les raisonnements.qui ont permis de l'établir le sont, peut-être, beaucoup moins.

Quoi qu'il en soit, les résultats sont à rapprocher de ceux qu'Heisenberg $\left({ }^{2}\right)$ a obtenus dans sa théorie désormais clas-

(1) J. de Physique [7], 1 (1930), 325.

(') Z. f. Physik, 49 (1928), 619. 
sique du ferromagnétisme. Il traite le cas d'un seul spin non compensé par atome et il trouve que le ferromagnétisme n'apparaît que lorsqu'un atome possède au moins 8 voisins. En adoptant le langage précédent, le facteur de réduction est égal à zéro quand le nombre des voisins est inférieur à 8 , puisqu'il n'y a pas d'orientation spontanée. Il est égal à I

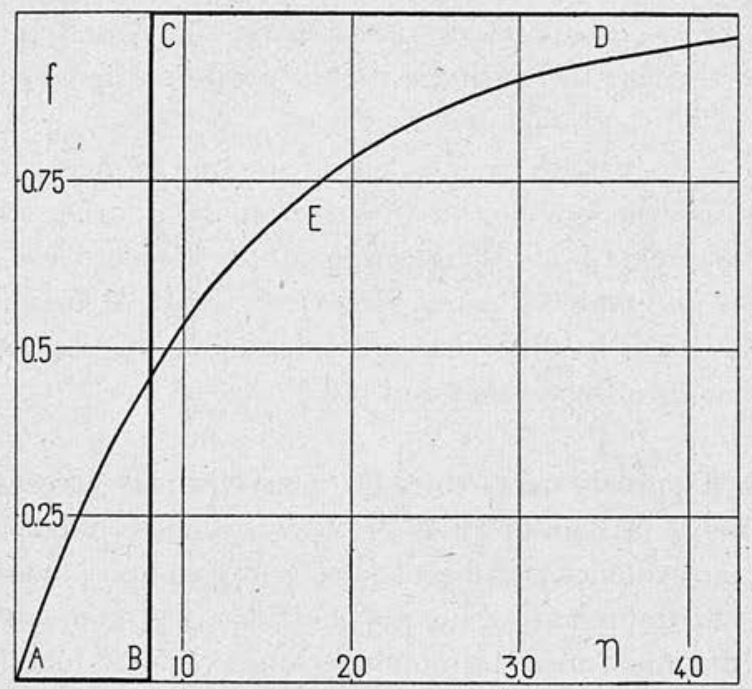

Fig. I . - Le facteur de réduction du moment ferromagnétique en fonction du nombre des voisins pour des atomes portant un seul spin. Quand il y a plusieurs spin sur un atome, $n$ représente le produit du nombre des spin par le nombre des voisins.

lorsque le nombre des voisins est égal, ou supérieur à 8 , puisque tous les moments sont orientés. Ces résultats sont traduits sur la figure I I par la ligne brisée $A B C D$, qui représente le facteur de réduction $f$, en fonction du nombre $n$ des voisins, pour un atome portant un seul spin non compensé D'autre part, j'ai représenté en E la courbe du 
facteur de réduction qui résulte de la théorie développée ici.

Le rapprochement des deux courbes fait supposer que la théorie de Heisenberg n'est qu'une première approximation.

Après la parution du mémoire de Heisenberg, différents auteurs ont repris la théorie du ferromagnétisme sous différentes formes ( $\left.{ }^{1}\right)$. Dans ces théories, d'une façon ou d'une autre, on en vient à supposer que le nombre des voisins est infini : dans ces conditions, on obtient évidemment la saturation totale.

Bref, les considérations développées ici ne paraissent pas en contradiction avec les théories admises actuellement mais les complèlent plutôt. Elles ont l'avantage de mettre nettement en évidence le rôle joué par les atomes voisins sur le moment d'un atome. D'après les données expérimentales, ce rôle est indéniable : c'est la conclusion à laquelle est arrivé Fallot $\left({ }^{2}\right)$ après étude des alliages de fer.

\$ 33. Appendice. - Calcul des moments atomiques des ferro-cobalts. - Supposons qu'en première approximation, la maille fondamentale du réseau reste de $2,86 \AA$, comme

TABLEAU XIII

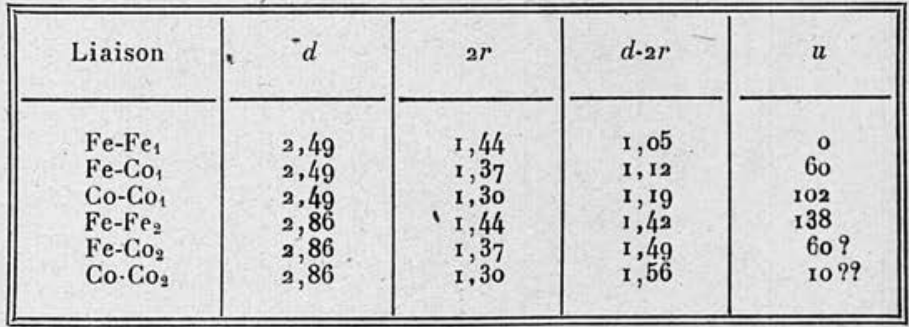

(1) Par exemple Slater. Phys. Rev., $34\left(19^{2} 9\right)$, I 293 . Voir aussi l'exposé de W. Pauli au Conseil Solvay de I930 (Paris, GauthierVillars, 1932), les théories de Bloch. Z. $f$. Pkysik, 52 (r $\left.9^{28}\right), 555$ et aussi $57\left(19^{2} 9\right), 545$.

(2) Thèse Strasbourg, 1935. 
dans le fer pur. Il ne faut tenir compte que des voisins situés sur les deux premières couches, c'est-à-dire à des distances de $2,49 \AA$ et $2,86 \AA$ de l'atome central. On a ainsi 6 catégories de liaisons distinctes dont le tableau XIII donne les caractéristiques et, en particulier, les énergies de liaison a correspondantes.

Toutes les liaisons sont à interaction positive, donc efficaces, sauf la liaison $\mathrm{Fe}-\mathrm{Fe}_{1}$.

Dans les ferro-cobalts, j'ai supposé, pour simplifier, que, lorsque la proportion de cobalt était égale ou inférieure à $50 \mathrm{o} / \mathrm{o}$, se produisaient 4 types de surstructures caractérisées par les proportions suivantes de cobalt : $\frac{1}{16}, \frac{1}{8}, \frac{1}{4}, \frac{1}{2}$. Ces surstructures sont représentées sur la figure $\mathbf{2}$.

Tableau XIV

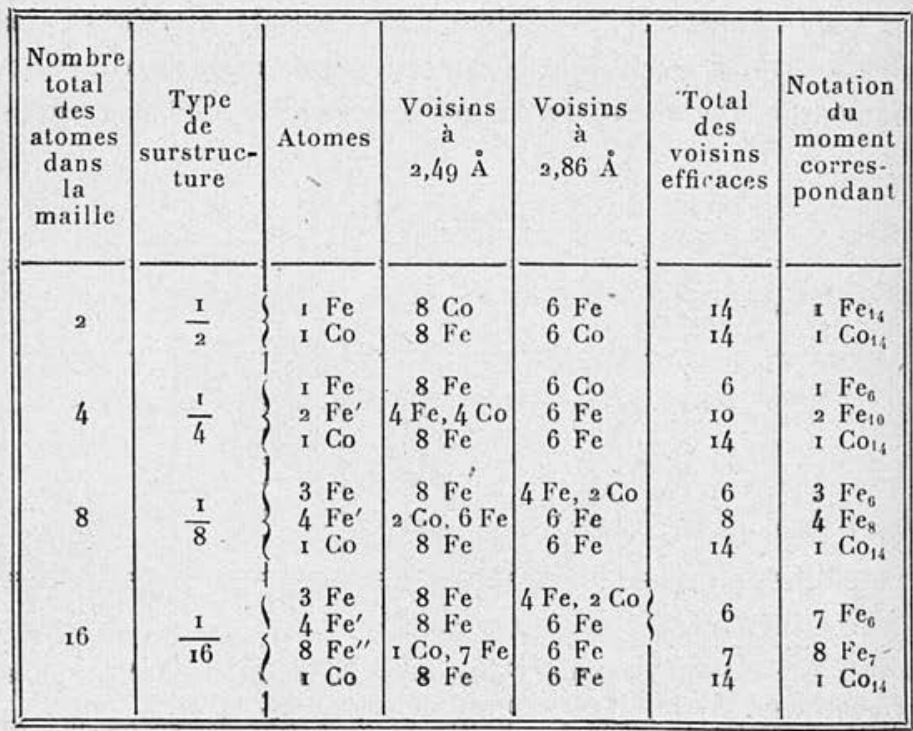


J'ai résumé dans le tableau XIV le type d'entourage de chaque atome. Dans certains cas, les atomes de fer ne sont pas tous identiques; je les ai distingués par des accents : $\mathrm{Fe}, \mathrm{Fe}^{\prime}, \mathrm{Fe}^{\prime \prime}$. Les chiffres qui précèdent ces notations indiquent le nombre des atomes de l'espèce considérée qui existent dans la maille élémentaire.

Pour chacune des surstructures considérées, le moment moyen $m$, par atome, est donné par les formules suivantes :

Surstructure à $\frac{\mathrm{I}}{2} \mathrm{Co} . \quad . \quad 2 m=\mathrm{Co}_{14}+\mathrm{Fe}_{14}$

Surstructure à $\frac{\mathrm{r}}{4} \mathrm{Co} . \quad . \quad 4 m=\mathrm{Co}_{14}+\mathrm{Fe}_{6}+{ }_{2} \mathrm{Fe}_{10}$

Surstructure à $\frac{\mathrm{r}}{8} \mathrm{Co} . \quad . \quad 8 m=\mathrm{Co}_{14}+3 \mathrm{Fe}_{6}+4 \mathrm{Fe}_{8}$

Surstructure à $\frac{1}{16} \mathrm{Co} . \quad . \quad{ }_{1} 6 m=\mathrm{Co}_{14}+{ }_{7} \mathrm{Fe}_{6}+8 \mathrm{Fe}_{7}$.

Avec la base $\mathrm{Ni}_{12}=3$, c'est-à-dire en prenant une seule probabilité d'occupation : celle du nickel, on calcule :

$m_{\frac{1}{2}}=\mathrm{II}, 60 ; \quad m_{\frac{1}{4}}=11,74 ; \quad m_{\frac{1}{8}}=11,59 ; \quad m_{\frac{1}{10}}=11,43$.

En tenant compte des deux probabilités d'occupation du cube centré, avec les bases $\mathrm{Fe}_{6}=\mathrm{II}_{1}, \mathrm{O}$ et $\mathrm{Fe}_{14}={ }_{14}, 6$, on calcule :

$m_{\frac{1}{2}}=11,85 ; \quad m_{\frac{1}{4}}=11,89 ; \quad m_{\frac{1}{8}}=11,64 ; \quad m_{\frac{1}{16}}=11,38$.

Ces valeurs ont été utilisées dans le tracé de la figure 9 .

Ferro-nickels $\alpha$. - Les moments à saturation des ferronickels (fig. ro) ont été calculés d'une manière entièrement analogue. Il y a une seule différence : la liaison $\mathrm{Ni}-\mathrm{Ni}_{2}$ à $2,86 \AA$ correspond à une interaction très probablement nulle tandis que l'énergie d'interaction de la liaison corres- 
pondante $\mathrm{Co}_{\mathrm{C}} \mathrm{Co}_{2}$ n'était pas nulle. Cela n'a d'ailleurs pas d'importance car cette liaison n'existe pas dans le domaine de concentrations dans lequel les ferro-nickels sont dans l'état $\alpha$. 